\title{
A Eutectic Dual-Phase Design towards Superior Mechanical Properties of Heusler-type Ferromagnetic Shape Memory Alloys
}

Zhigang $\mathrm{Wu}^{1,2^{*}}$, Zhiwen Liang ${ }^{1}$, Yajiu Zhang ${ }^{1}$, Zhuhong $\mathrm{Liu}^{3}$, Junsong Zhang ${ }^{2}$, Fakhrodin Motazedian $^{2}$, Sam Bakhtiari², Bashir Samsam Shariat ${ }^{2}$, Yinong Liu²*, Yang Ren ${ }^{4}$ and Hong Yang $^{2}$

${ }^{1}$ School of Civil Engineering, Guangzhou University, Guangzhou 510006, China

${ }^{2}$ Department of Mechanical Engineering, The University of Western Australia, Perth, WA 6009, Australia

${ }^{3}$ Department of Physics, University of Science and Technology Beijing, Beijing 100083, China

${ }^{4}$ X-ray Science Division, Argonne National Laboratory, Argonne, IL 60439, USA

Corresponding author: Dr Zhigang Wu: gordon.wu@uwa.edu.au;

Corresponding author: Prof. Yinong Liu. Email: yinong.liu@uwa.edu.au

\begin{abstract}
Heusler-type ferromagnetic shape memory alloys possess attractive multifunctional properties, including magnetic field induced shape memory effect, magnetoresistance and magnetocaloric effect, owing to the unique concurrent magnetic and martensitic transformations. However, these intermetallics generally exhibit intrinsic high brittleness and low strength, which severely impede their workability for processing and applicability in real use. In this study, we demonstrate a new grain refining strategy by means of eutectic solidification to improve the mechanical properties in Ni-Mn-Sn-Fe alloys. In a fully eutectic microstructure, the average $\gamma$ lamellae thickness was refined to $\sim 170 \mathrm{~nm}$ and the composite showed a compressive strength of $1950 \mathrm{MPa}$, ductility of 19.5\%, Young's Modulus of $38 \mathrm{GPa}$, pseudoelasticity of 3.2\% and high mechanical cyclic stability. The high mechanical performance is attributed to the effect of departmentalisation of the brittle Heusler alloy by the densely distributed $\gamma$ phase fine lamellae in resisting crack propagation. The eutectic Heusler composite exhibited a metamagnetic phase transformation, with a magnetic entropy change of $10.2 \mathrm{~J} / \mathrm{kg} \cdot \mathrm{K}$ and a refrigeration capacity of $181 \mathrm{~J} / \mathrm{kg}$ in a field change of $5 \mathrm{~T}$.
\end{abstract}

Keywords: Heusler alloys; Ductility; Eutectic microstructure; Grain refining; Magnetic entropy change 


\section{Introduction}

Heusler-type ferromagnetic shape memory alloys (FSMAs) have been extensively studied since Ullakko et al. first reported a magnetic-field-induced strain (MFIS) of $0.2 \%$ in a $\mathrm{Ni}_{2} \mathrm{MnGa}$ Heusler alloy via a martensite variant reorientation process in 1996 [1]. Since then, there has been a progressive and remarkable improvement of the MFIS to $10-12 \%$ in the same mechanism [2, 3], drastically enhancing their potential for practical applications. However, the low blocking stresses for the magnetic-field-induced variant reorientation in Ni-Mn-Ga alloys, typically in the range of 2-6 $\mathrm{MPa}[4,5]$, make them impractical for meaningful actuation applications [6]. For this, Ni-Co-Mn-X (X=In, Sn, Sb) alloys have gained much attention in recent years due to their ability to yield higher work outputs with stresses of $\sim 100 \mathrm{MPa}$ and large recoverable strains of $3 \%$ under a magnetic field of a few Tesla [7, 8]. Such high magnetomechanical performance is associated with a concurrent magnetic and structural transformation (i.e. a metamagnetic martensitic transformation), from a pre-deformed weakly magnetic martensite to a strongly ferromagnetic austenite. Accompanying the magnetomechancal effect, significant magnetocaloric effect and magnetoresistance effect have also been found in Ni-Co-Mn-X (X=In, Sn, Sb) Heusler alloys. For example, an adiabatic temperature change of $6.2 \mathrm{~K}$ was measured in a Ni45.2Mn36.7In13C05.1 alloy associated with a reverse martensitic transformation induced by a $1.9 \mathrm{~T}$ magnetic field [9]. In another case, a magnetoresistance increase of greater than $50 \%$ was measured in a $\mathrm{Ni}_{50} \mathrm{Mn}_{36} \mathrm{Sn}_{14}$ alloy in magnetic fields up to $17 \mathrm{~T}$ [10]. In addition to the magnetocaloric and magnetoresistance effects, Heusler-type FSMAs have also shown attractive elastocaloric effect associated with the stress-induced martensitic transformation. For example, a temperature change of $3.5 \mathrm{~K}$ was realized in a Ni45.7Mn36.6 In13.3 $\mathrm{C}_{5.1}$ polycrystal under $100 \mathrm{MPa}$ [11] and a temperature change of $5.5 \mathrm{~K}$ was recorded in a $\mathrm{Ni}_{43.5} \mathrm{Mn}_{39} \mathrm{Sn}_{11} \mathrm{Co} .5$ polycrystal under $310 \mathrm{MPa}$ [12]. The above novel physical properties make Heusler-type FSMAs a versatile class of functional materials.

A severe drawback of NiMn-based FSMAs is the intrinsic brittleness (thus low strength), making them vulnerable in design and jeopardizing their advantageous functional properties for practical applications [13]. The brittleness of these alloys originates from two causes: (i) intrinsic brittleness of the crystal structure and (ii) transformation volume change. Heusler alloys are highly ordered intermetallics which are intrinsically brittle due to the low tolerance for dislocation movement. The gliding of one dislocation across a crystallographic plane in such ordered structures brings like atoms into contact and breaks the ordering of the structure, and it takes another (or more) dislocation to glide through the same path to restore the original structure. In other words, the Burger's vector of a full dislocation in a L2 1 Heusler structure is in principle four times of the Burger's vector of a full dislocation in its disordered bcc counterpart structure. The lack of easy mechanism for plasticity causes the Heusler single crystals to crack via transgranular cleavage under load. The martensitic transformations in Heusler alloys normally show molar volume reductions of $0.3-1.2 \%$ [14-16]. Such volume changes cause hydrostatic stresses within the structure and intergranular cracking along grain boundaries. Intergranular cracking is a prevalent problem for polycrystalline Heusler alloys, preventing them to be used in real applications, despite their low cost relative to single crystals. Thus, reducing the brittleness of polycrystalline NiMn-based Heusler alloys has been a focus of many studies. 
The most common approach to reducing the brittleness of these alloys is to introduce a ductile fcc second phase ( $\gamma$ phase), which is effective in hindering crack propagation in the Heusler matrix [24]. This is achieved by either increasing the Ni content to above the stoichiometry of 50 at\% [17] or introducing a quaternary substitutional element for $\mathrm{Mn}$ or the main group element X in the Ni-Mn-X structure, such as Fe [18, 19], Co [20, 21], Cr [22], V [23] and Cu [24]. This approach has shown clear improvement to the mechanical properties. For example, Tan et al. reported that the compressive strength of a Ni-Mn-Sn Heusler alloy increased from $225 \mathrm{MPa}$ to $725 \mathrm{MPa}$ and its ductility increased from $4.9 \%$ to $9.3 \%$ by the addition of 4 at\% Fe [25]. The volume fraction of the $\gamma$ phase has been found to increase with higher contents of transition elements, and a higher fraction of the $\gamma$ phase generally gives higher strength and ductility to the Heusler composites [24, 26]. Using this approach, Ma et al demonstrated the ability to hot roll a $\mathrm{Ni}_{56} \mathrm{Mn}_{25-\mathrm{y}} \mathrm{Co}_{\mathrm{y}} \mathrm{Ga}_{19}(\mathrm{y}=4,8)$ alloy cast buttons into $\sim 0.5 \mathrm{~mm}$ thin plates and conducted tensile testing on them [26]. They reported a tensile strength $729 \mathrm{MPa}$ and a tensile elongation of $14.1 \%$ in an alloy with $y=8$ and a $\gamma$ phase fraction of $43 \%$. Yang et al. studied the effectiveness of different substitutional elements on ductility improvement of $\mathrm{Ni}_{56} \mathrm{Mn}_{25}$ $\mathrm{x}_{\mathrm{x}} \mathrm{Ga} 19(\mathrm{Z}=\mathrm{Fe}, \mathrm{Co}$ and $\mathrm{Cu})$ alloys, and found that the effectiveness decreases in the order of $\mathrm{Fe}, \mathrm{Co}$ and $\mathrm{Cu}$ [27]. The superelastic cycles of Heusler alloys can also be significantly increased and thus the functional fatigue life of Heusler alloys, by modifying the single phase microstructure to a dual-phase microstructure. For example, a single phase $\mathrm{Ni}_{54} \mathrm{Fe}_{19} \mathrm{Ga}_{27}$ alloy showed a temperature change of $4 \mathrm{~K}$ under $170 \mathrm{MPa}$ but failed after only 10 superelastic cycles, while the dual-phase microstructure that was obtained after annealing at $1273 \mathrm{~K}$ for 3 hours could withstand 100 loading-unloading cycles with negligible degradation of the elastocaloric effect [28].

In general, the previously reported dual-phase Heusler alloys have two main drawbacks. First, transgranular cleavage cracking still occurs, though largely reduced [29]. This is particularly prevalent in microstructures with large spacing between the $\gamma$ phase grains $[29,30]$. This implies that the $\gamma$ phase spacing needs to be reduced to limit the space for crack propagation in the brittle Heusler phase. Second, the shape memory ability is often compromised by the presence of the $\gamma$ phase [31,32]. The $\gamma$ phase exhibits no martensitic transformation and deforms via dislocation slip [33], thus acts as a pure mechanical resistance and weakens the alloy's ability for shape recovery. For example, the shape memory effect almost vanished in a $\mathrm{Ni}_{56} \mathrm{Mn}_{17} \mathrm{Co}_{8} \mathrm{Ga}_{19}$ dual-phase alloy with a $\gamma$ phase volume fraction of $43 \%$ [26].

For mechanical property optimisation, effort has largely been focused on volume fraction of the $\gamma$ phase, and little attention has been given to its morphology in the Heusler matrix, including the size, shape and distribution. The grain size of the $\gamma$ phase in the reported Heusler composites is typically $3 \sim 30 \mu \mathrm{m}$ following traditional solidification and solution heat treatments [30, 33]. Sui et al. added element Y to Ni-Mn-Ga alloys and obtained a hypoeutectic structure in sub-micron scale [34]. However, the second phase formed is an ordered hexagonal $\mathrm{Y}(\mathrm{Ni}, \mathrm{Mn})_{4} \mathrm{Ga}$ compound which is also brittle in nature, and increase of $\mathrm{Y}(\mathrm{Ni}, \mathrm{Mn})_{4} \mathrm{Ga}$ fraction was shown to deteriorate the strength and ductility of the composite. Up to date, grain 
refinement of ductile $\gamma$ phase and control of its distribution have not been reported in the literature.

Grain refinement of the $\gamma$ phase may have the benefit of reducing its hindrance to the reversible martensitic transformation and shape memory effect of the Heusler phase. At the lattice scale, dislocation slip in the $\gamma$ phase is highly inhomogeneous [35] and does not match well with the uniform lattice distortion of the martensite [36]. This is the origin of the mechanical resistance of the $\gamma$ phase to the shape memory effect of the alloy. Such adverse effect may be much reduced if the $\gamma$ phase can deform largely elastically along with the martensitic transformation, and reducing the grain size of the $\gamma$ phase to nanoscale will be helpful. Recently, a NiTi/Nb nanowire composite has been shown to be able to induce ultra-large elastic strains of $6 \%$ in the $\mathrm{Nb}$ nanowires embedded in the NiTi matrix [37]. This is explained on the basis of a novel concept of elastic and inelastic (transformation) strain matching between the $\mathrm{Nb}$ nanowires and NiTi martensitic matrix at the atomic scale. The large elastic strain ( 6\%) obtained in the Nb nanowires is closely related to their small dimensions, in which generation and storage of dislocations are largely prevented [38, 39]. Therefore, by reducing the grain size of the $\gamma$ phase, it is possible to achieve higher elastic strains, thus to have a better matching with the lattice distortion during the martensitic transformation of the Heusler alloy.

Obtaining both fine grain size and interspacing of the toughening phase in Heusler alloys seems difficult and cannot be realised in the reported dual-phase Heusler systems. In this study, we report a new strategy of morphology control in Heusler alloys to produce an ultrafine Heusler/ $\gamma$ dual-phase microstructure by eutectic solidification. In order to achieve the eutectic microstructure, the data of existing binary and ternary phase diagrams were used to predict the eutectic phase formation. According to the ternary phase diagram of Ni-Mn-Sn [40], the L21 structure is stable in a wide composition range of $\mathrm{Ni}_{50} \mathrm{Mn}_{25+\mathrm{x}} \mathrm{Sn}_{25-\mathrm{x}}(0 \leq \mathrm{x} \leq 15)$ which can be taken as the integrated pseudo-element for the eutectic reaction. The next step is to search for a doping element which can induce the formation of a $\gamma$ phase as the second integrated pseudoelement of the eutectic reaction. We accessed the binary phase diagrams of Fe-Ni [41], Fe-Mn [42] and Ni-Mn [43], and found all of them have a large fcc solid solution phase region, thus the high likelihood of forming $\gamma$ phase when Fe is added to Ni-Mn-Sn system. Furthermore, Fe-Ni [41], Fe-Mn [42] and Fe-Sn [44] all exhibit eutectoid/eutectic reactions. Hence, adding Fe to the Ni-Mn-Sn system provides a promising solution for creating the desired eutectic microstructure. Using this approach, we are able to achieve high strength, good ductility, stable mechanical cyclability and moderate superelasticity in these new eutectic Heusler $\gamma \gamma$ phase composites.

\section{Experimental methods}

Polycrystalline $\mathrm{Ni}_{50} \mathrm{Mn}_{40-\mathrm{x}} \mathrm{Sn}_{10} \mathrm{Fe}_{\mathrm{x}}(\mathrm{x}=3,4,5,6,7,8,9$ and 10) alloy ingots of approximately $10 \mathrm{~g}$ were prepared by means of arc melting in an argon atmosphere using high purity elemental $\mathrm{Ni}, \mathrm{Mn}, \mathrm{Sn}$ and Fe. The samples are designated as Fe3 to Fe10 based on the atomic percentage of Fe in the nominal compositions. Samples with various sizes were sectioned from the centre region of the cast ingots using a low speed diamond saw and a spark wire cutting machine for different examinations. Microstructure, chemical composition and fractographic analysis of the 
alloys were characterised by means of scanning electron microscopy (SEM) using a FEI Verios 460 instrument equipped with an X-ray energy dispersive spectrometry (EDS) and AZTEC software capability. The EDS composition quantification was calibrated using standard pure elements to achieve accuracy within $\pm 0.5 \%$. X-ray diffraction (XRD) was performed using a Panalytical Empyrean XRD instrument with $\mathrm{Cu}-\mathrm{K} \alpha$ radiation to characterise the phase formation in the alloys. Transformation behaviour of the alloys was analysed by means of differential scanning calorimetry (DSC) using a TA Q10 DSC in $\mathrm{N}_{2}$ atmosphere with a cooling/heating rate of $10 \mathrm{~K} / \mathrm{min}$.

Compression testing of $3 \times 3 \times 5 \mathrm{~mm}$ blocks was performed using an Instron 5982 universal testing machine at room temperature with a strain rate of $3.33 \times 10^{-4} \mathrm{~s}^{-1}$. The thermomagnetic behaviour of the alloys was analysed using a MPMS-07 superconducting quantum interference device magnetometer (SQUID), in a small field of 100 Oe with a cooling/heating rate of 3 $\mathrm{K} / \mathrm{min}$. To derive the magnetic entropy change $\left(\Delta \mathrm{S}_{\mathrm{M}}\right)$ at the magnetomartensitic transformation with thermal hysteresis, the so-called loop process [45] method was adopted to obtain the isothermal magnetisation curves ( $\mathrm{M}-\mathrm{H}$ curves). The $\mathrm{M}-\mathrm{H}$ curves were measured in magnetic fields up to $5 \mathrm{~T}$ upon heating with a temperature interval of $2 \mathrm{~K}$. Prior to each isothermal magnetisation, the sample was cooled down to $10 \mathrm{~K}$ to a complete martensite state to eliminate the history-dependent magnetic state and then heated to the target temperature for the magnetisation measurement. The $\Delta \mathrm{S}_{\mathrm{M}}$ was then derived from the resulting magnetisation curves using the Maxwell relation [46]: $\Delta \mathrm{S}_{M}=\int_{0}^{H}\left(\frac{\partial M}{\partial T}\right)_{H} d H$.

In-situ synchrotron high-energy X-ray diffraction (HE-XRD) measurements were performed during compression on beamline 11-ID-C at the Advanced Photon Source, Argonne National Laboratory, USA. The high-energy X-rays had a wavelength of $0.1173 \AA$ and a beam size of $0.6 \times 0.6 \mathrm{~mm}^{2}$. The diffraction was recorded in the transmission geometry using a PerkinElmer large area two-dimensional (2D) detector. One-dimensional (1D) HE-XRD diffraction spectrums were obtained by integrating the 2D diffraction pattern along the longitudinal direction (loading direction) and along the entire Debye ring (0-360 azimuth angle). Gaussian fits were employed to determine the positions of the diffraction peaks for d-spacing and lattice parameter measurements. The lattice $d$-spacing strains for a particular set of planes is calculated as $\varepsilon_{h k l}=\left|d_{h k l}-d_{h k l}^{0}\right| / d_{h k l}^{0}$, where $d_{h k l}^{0}$ is the peak position at zero applied stress. The errors of the lattice strain measurements and relative peak intensity measurements were estimated to be less than $0.05 \%$.

\section{Results}

\subsection{Alloy microstructure and phase identification}

Figure 1 shows back-scattered SEM micrographs of Fe3-Fe11 samples in their as-cast state. $\mathrm{Fe} 3$ and Fe4 (Figures 1(a) and 1(b), respectively) exhibited a single phase microstructure, with the presence of some original intergranular cracks. These cracks are caused by the volume change of the martensitic transformation. Fe5 had a hypoeutectic microstructure (Figure 1(c)), with a large volume fraction of the pre-eutectic Heusler phase in dendritic formation (light 
contrast) and a small fraction of the $\gamma$ phase lamellae (dark contrast) in gaps among the large Heusler dendrites. The Heusler dendrites show severe chemical segregation, as evidenced by the clear light grey contrast regions (arrowed). The volume fraction of the eutectic component increased with increasing the Fe content, as seen in Fe6 and Fe7 (Figures 1(d) and (e), respectively). The alloy is practically fully eutectic at 8 at\% Fe (Figure 1(f)). Fe9, Fe10 and Fe11 all showed a hypereutectic microstructure (Figures 1(g), (h) and (i), respectively), with progressively increasing amount of the pre-eutectic $\gamma$ phase. It is apparent that the eutectic microstructure in Fe8 has the finest and most uniform distribution of the $\gamma$ phase among the alloys.

The as-solidified Fe8 alloy showed colonies of eutectic lamellae enveloped by a non-planar growth front of a dendritic-like morphology (dashed lines in (f)). The dendrite-like eutectic morphology is indicative of incongruent eutectic solidification caused by segregation and is commonly observed in melts containing complex and high alloying elements [47]. The dendrite-like colony structure also showed finer lamellae in the centre zone and a much coarser morphology in the boundary zone. It is known that the lamellae tend to grow normal to the solid-liquid interface. The dashed line transverse across the colonies gives an indication of the shape of the solid-liquid interface from which the dendrites originated. With the growth of the eutectic dendrites, the interface line (dendrite tip) becomes longer and the lamellae become more separated. The increased distance for solute to diffuse into each lamellae may have caused the reduced growth rate and enlarged thickness at the dendritic boundaries [48].

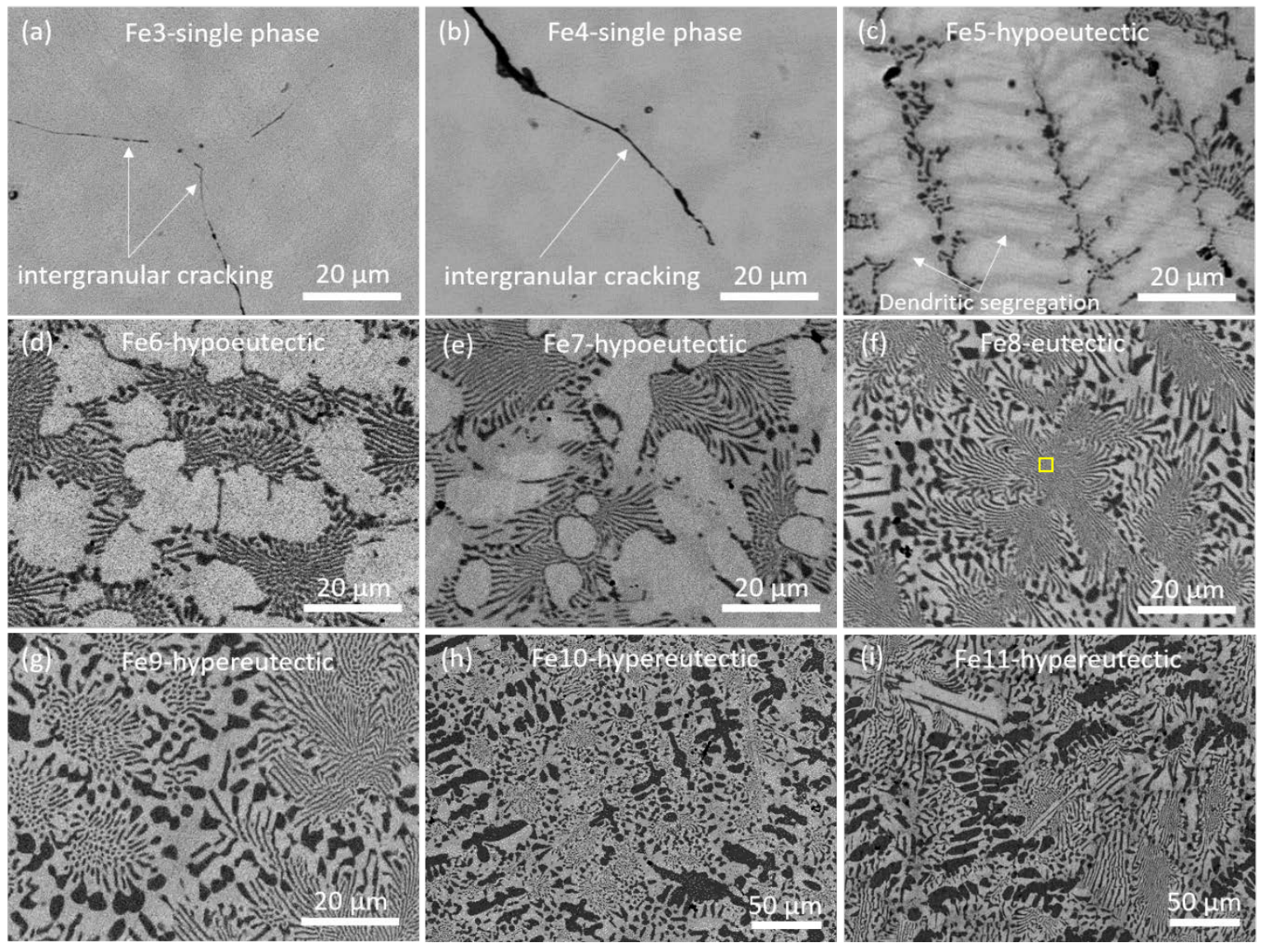


Fig. 1. Back-scattered electron micrographs of $\mathrm{Ni}_{50} \mathrm{Mn}_{40-\mathrm{x}} \mathrm{Sn}_{10} \mathrm{Fe}_{\mathrm{x}}$ alloys. The phase in the light contrast is the Heusler phase and that in the dark contrast is the $\gamma$ phase. Micrographs (a)-(g) were taken at 3000× and micrographs (h)-(i) were taken at 500×.

Figure 2 shows TEM examination and EDS analysis of the eutectic Fe8 alloy. Figure 2(a) is a bright field TEM image of the eutectic structure, taken from the central region of a eutectic colony, as indicated by the yellow box shown in Figure 1(f). The $\gamma$ lamellae, which are in the bright contrast, are approximately $150 \mathrm{~nm}$ in thickness and $400 \mathrm{~nm}$ in spacing between them. The insets in Figure 2(a) show the selected area electron diffraction (SAED) patterns of the ordered L2 1 Heusler phase (the upper inset) and fcc $\gamma$ phase (the lower inset) confirming their phase identification. Figure 2(b) shows a HADDF TEM micrograph and (c), (d), (e) and (f) are the elemental mapping images for $\mathrm{Ni}, \mathrm{Mn}, \mathrm{Sn}$ and $\mathrm{Fe}$, respectively. It is seen that the $\gamma$ phase is much richer in Fe content and poorer in Sn compared to the Heusler phase. The differences in $\mathrm{Ni}$ and $\mathrm{Mn}$ contents are relatively small between the two phases.

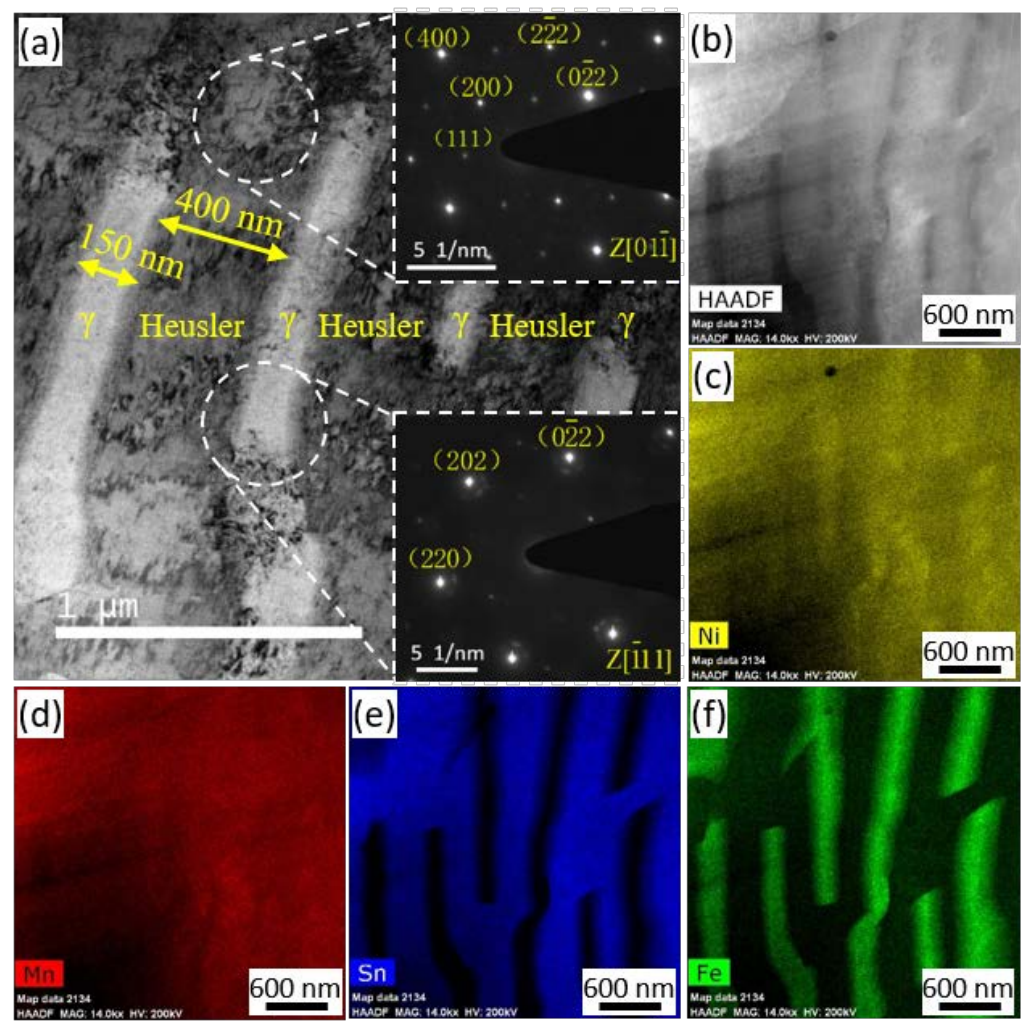

Fig. 2. TEM examination and EDS elemental analysis of Fe8 alloy. (a) TEM bright field micrograph. (b) HADDF micrograph, and its EDS mapping for (c) Ni, (d) Mn, (e) Sn and (f) Fe.

Compositions of the Heusler phase and the $\gamma$ phase were determined by EDS analysis and the results are summarised in Table 1 . The total volume fraction of the $\gamma$ phase (including both the pre-eutectic and eutectic $\gamma$ phases) and the average $\gamma$ lamellae thickness and lamellar spacing of the eutectic were determined from SEM micrographs using ImageJ and the results are also presented in Table 1. It is seen that the $\gamma$ phase is largely a Ni-Mn-Fe phase, with a low Sn content. On the other hand, the Heusler phase is a Ni-Mn-Sn phase, with a low Fe content. The formation of the $\gamma$ phase inevitably altered the composition of the Heusler phase. With the 
substitution of Fe for Mn, the Heusler phase showed a decrease in Mn content and increase in Sn content, and small variations in Ni content.

Table 1. Chemical compositions of the Heusler and $\gamma$ phases, e/a ratio of the Heusler phase, average $\gamma$ lamellae thickness and lamellar spacing, and total volume fraction of the $\gamma$ phase.

\begin{tabular}{l|cccc|ccccc|c|c|c}
\hline Alloy & \multicolumn{4}{|c|}{ Heusler phase } & \multicolumn{6}{c}{ Eutectic $\gamma$ phase } & $\begin{array}{c}\text { Vol\% } \\
\gamma\end{array}$ \\
& $\begin{array}{c}\text { Ni } \\
\text { (at\%) }\end{array}$ & $\begin{array}{c}\text { Mn } \\
\text { (at\%) }\end{array}$ & $\begin{array}{c}\text { Sn } \\
\text { (at\%) }\end{array}$ & $\begin{array}{c}\text { Fe } \\
\text { (at\%) }\end{array}$ & $\begin{array}{c}\text { e/a } \\
\text { ratio }\end{array}$ & $\begin{array}{c}\text { Ni } \\
\text { (at\%) }\end{array}$ & $\begin{array}{c}\text { Mn } \\
\text { (at\%) }\end{array}$ & $\begin{array}{c}\text { Sn } \\
\text { (at\%) })\end{array}$ & $\begin{array}{c}\text { Fe } \\
\text { (at\%) }\end{array}$ & $\begin{array}{c}\text { Lamellae } \\
\text { thickness } \\
(\mu \mathrm{m})\end{array}$ & $\begin{array}{c}\text { Lamellar } \\
\text { spacing } \\
(\mu \mathrm{m})\end{array}$ & \\
\hline Fe3 & 50.0 & 37.4 & 10.1 & 2.5 & 8.222 & - & - & - & - & - & - & - \\
Fe4 & 49.9 & 36.4 & 10.5 & 3.2 & 8.214 & - & - & - & - & - & - & - \\
Fe5 & 49.2 & 36.2 & 11.3 & 3.3 & 8.170 & 51.0 & 33.7 & 1.6 & 13.7 & 0.41 & 1.36 & 12.1 \\
Fe6 & 49.3 & 35.2 & 12.0 & 3.5 & 8.154 & 51.5 & 33.2 & 1.5 & 13.8 & 0.33 & 1.10 & 20.7 \\
Fe7 & 49.2 & 34.6 & 12.5 & 3.7 & 8.138 & 51.1 & 33.5 & 1.5 & 13.9 & 0.25 & 0.83 & 25.2 \\
Fe8 & 49.1 & 33.7 & 13.4 & 3.8 & 8.109 & 51.9 & 31.9 & 1.4 & 14.8 & 0.17 & 0.56 & 30.4 \\
Fe9 & 48.5 & 32.5 & 15.1 & 3.9 & 8.041 & 51.5 & 31.8 & 1.6 & 15.1 & 0.23 & 0.77 & 34.9 \\
Fe10 & 48.9 & 30.6 & 16.5 & 4.0 & 8.012 & 50.2 & 29.6 & 2.6 & 17.6 & 0.53 & 1.76 & 41.4 \\
Fe11 & 48.1 & 31.1 & 16.8 & 4.0 & 7.979 & 50.1 & 28.8 & 2.8 & 18.3 & 0.96 & 3.20 & 47.1 \\
\hline
\end{tabular}

The change of composition of the Heusler phase alters the transformation behaviour. To evaluate this influence, the e/a ratio of the Heusler phase is calculated. Figure 3(a) shows the effects of Fe content on the e/a ratio and eutectic structure of the alloys. It is seen that the e/a ratio decreased from 8.222 in Fe3 to 7.979 in Fe11. This is largely due to the increase of Sn content in the Heusler phase, which has fewer valence electrons (4), and the decrease of Mn, which has a higher number of valence electrons (7). A decrease in e/a ratio may also imply lowering of the martensitic transformation temperature [49]. In the figure, the Fe content is divided into three regions based on the microstructure of the alloys, including single Heusler phase region at $\leq 4$ at $\% \mathrm{Fe}$, hypoeutectic region at $4 \sim 8$ at $\% \mathrm{Fe}$, and hypereutectic region at $>8$ at $\% \mathrm{Fe}$.

Figure 3(b) shows the effect of Fe content on the total volume fraction of the $\gamma$ phase and the average $\gamma$ phase lamellae thickness in the eutectic. The total volume fraction of the $\gamma$ phase increased progressively from $12 \%$ to $47 \%$ with increasing the Fe content from 3 at $\%$ to 11 at $\%$. The average thickness of the eutectic $\gamma$ phase lamellae varied between 170 and $960 \mathrm{~nm}$. Apparently, Fe8 alloy has the finest microstructure among all alloys. 

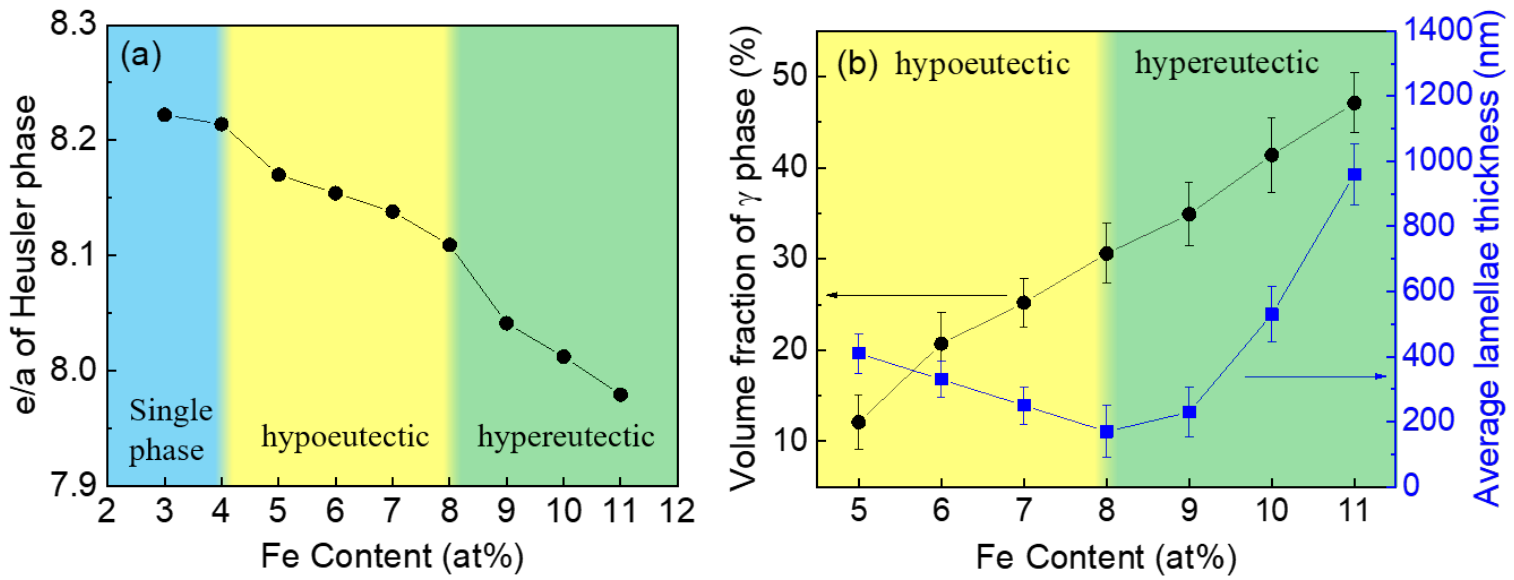

Fig. 3. Effect of Fe content on the e/a ratio of the Heusler phase and characteristics of the $\gamma$ phase of $\mathrm{Ni}_{50} \mathrm{Mn}_{40-\mathrm{x}} \mathrm{Sn}_{10} \mathrm{Fe}_{\mathrm{x}}$ alloys. (a) e/a ratio of the Heusler phase. (b) Volume fraction of the total $\gamma$ phase and average thickness of $\gamma$ phase lamellae.

\subsection{Crystal structure}

Figure 4 shows the XRD spectrums of the alloys at room temperature. $\mathrm{Fe} 3, \mathrm{Fe} 4$ and $\mathrm{Fe} 5$ showed a single phase 40 martensite structure, which is consistent with the previously reported NiMn-Sn alloys [50, 51]. Fe6 showed a mixed structure of the L 21 austenite, $4 \mathrm{O}$ martensite and fcc $\gamma$ phase. At higher Fe contents, the alloys showed L2 1 austenite and fcc $\gamma$ phase without the martensite, indicating the suppression of the martensitic transformation temperature by $\mathrm{Fe}$ addition in these alloys. This is due to the decrease of e/a ratio of the Heusler phase with the increase of Fe content (Figure 3(a)). 


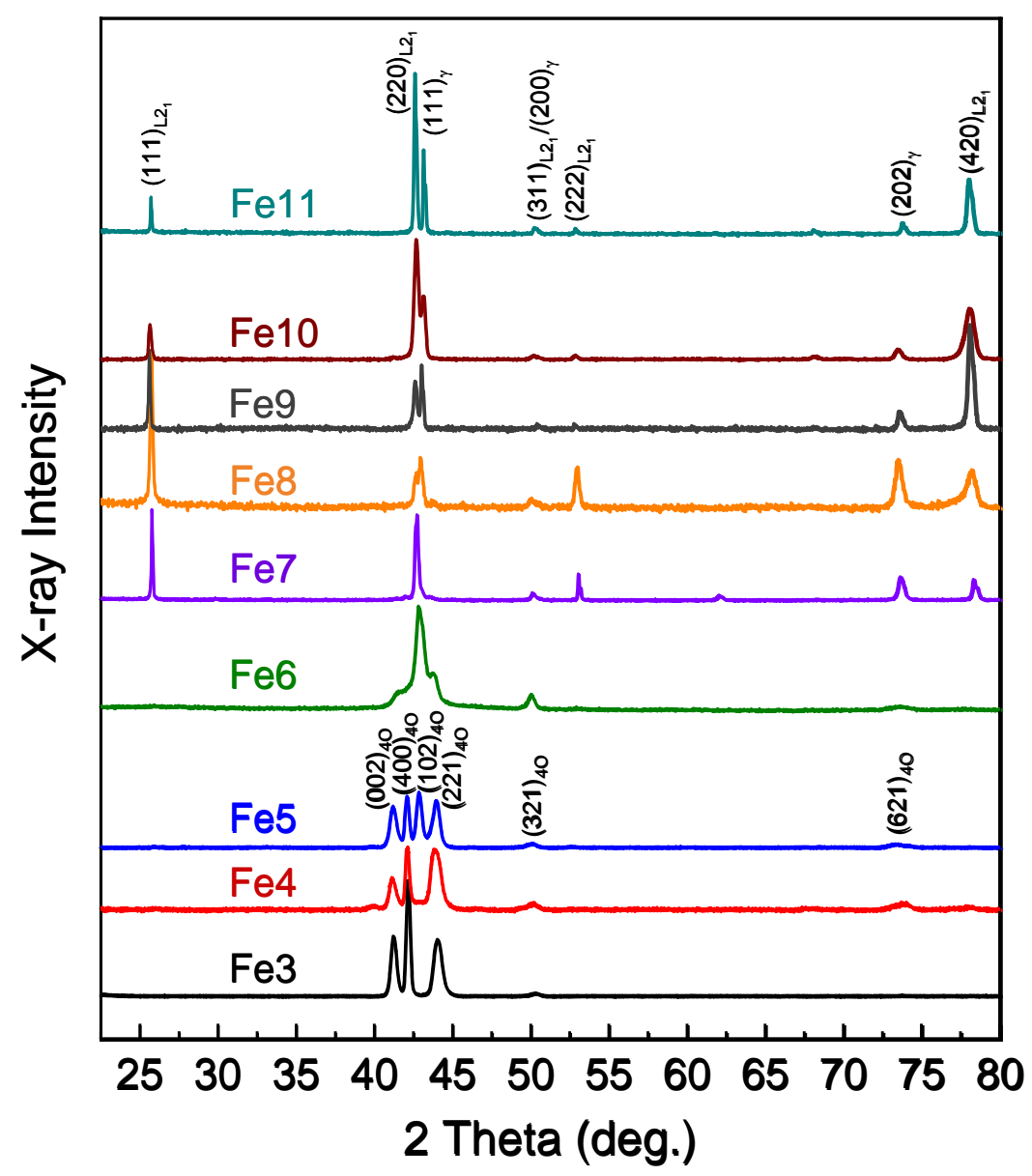

Fig. 4. X-ray diffraction spectrums of $\mathrm{Ni}_{50} \mathrm{Mn}_{40-\mathrm{x}} \mathrm{Sn}_{10} \mathrm{Fe}_{\mathrm{x}}$ alloys at room temperature.

Figure 5 shows the lattice constants and unit cell volume determined from the XRD measurement as functions of e/a ratio of the Heusler phase. The lattice constants of the 40 martensite and L2 1 austenite all decreased with increasing the e/a ratio, which leads to the decrease of unit cell volume for both the martensite $\left(V_{M}\right)$ and the austenite $\left(V_{A}\right)$. The transformation volume change $\Delta \mathrm{V}$ (volume contraction upon the forward transformation) is estimated to be $0.56 \%$, and appears to be independent of the Fe content. 


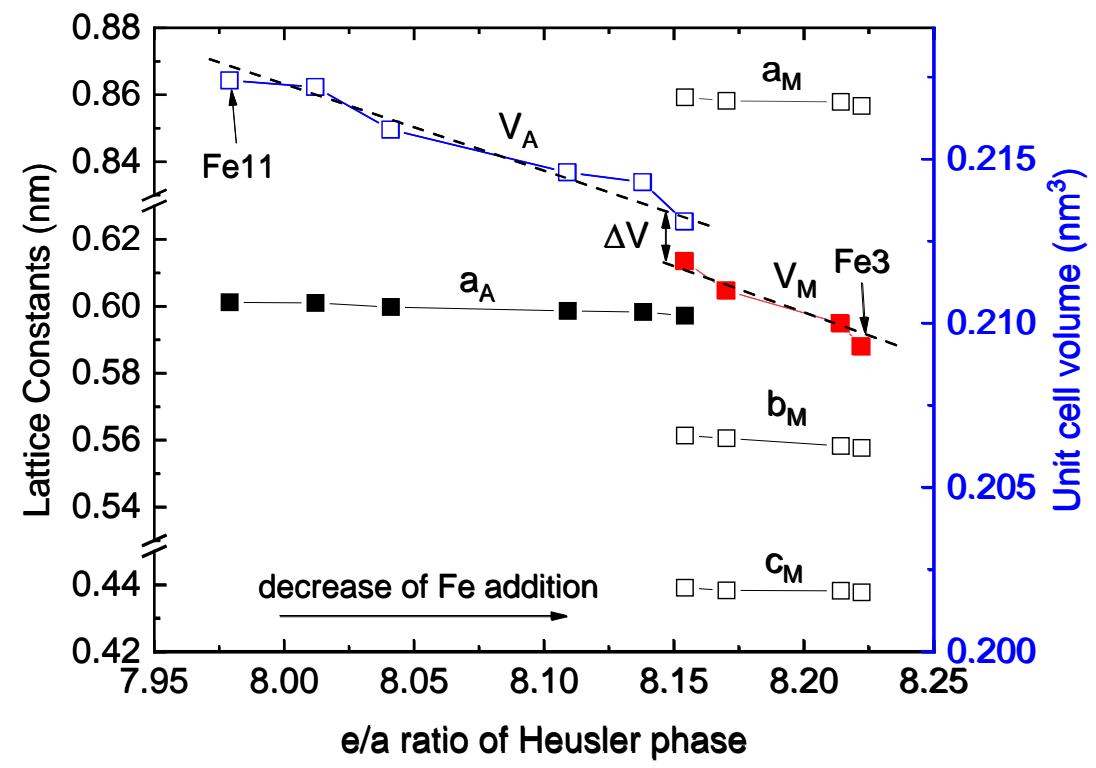

Fig. 5. Effect of e/a ratio on the lattice constants and unit cell volumes of the L21 austenite and $4 \mathrm{O}$ martensite in the $\mathrm{Ni}_{50} \mathrm{Mn}_{40-\mathrm{x}} \mathrm{Sn}_{10} \mathrm{Fe}_{\mathrm{x}}$ alloys.

\subsection{Alloy strength, ductility and mechanical cyclability}

Figure 6 presents the mechanical properties of the alloys. Figure 6(a) shows the compressive engineering stress-strain curves of Fe3-Fe11 samples. The early "yielding” (indicated by the arrows) of $\mathrm{Fe} 3, \mathrm{Fe} 4$ and $\mathrm{Fe} 5$ corresponds to the stress induced martensite reorientation, as these alloys are predominantly at a martensitic state at room temperature (Figure 4). Figure 6(b) shows the fracture strength and ductility as functions of the Fe content. The fracture strength increased from 770 to $1950 \mathrm{MPa}$ by increasing the Fe content from 3 to 8 at\%, and then decreased slightly with further increase of the Fe content. The increase of strength is attributed to the refinement of the microstructure, which is more resistive to crack formation and propagation due to its high density of inter-phase interfaces. For the hyper-eutectic alloys, the microstructure coarsened and the interface density decreased, causing decrease of the strength. The ductility showed a monotonic increase from $7.7 \%$ to $21.3 \%$ with increasing the Fe content from 3 to 11 at\%. Fe8 and Fe9, which had a nearly full eutectic structure, exhibited the best mechanical performance in both strength and ductility. It is also noted that the strain hardening behaviour in Fe10 and Fe11 are relatively weaker than that in Fe8 and Fe9, which may be due to their larger sizes of the pre-eutectic $\gamma$ phase allowing for relatively easier dislocation movement during deformation. 

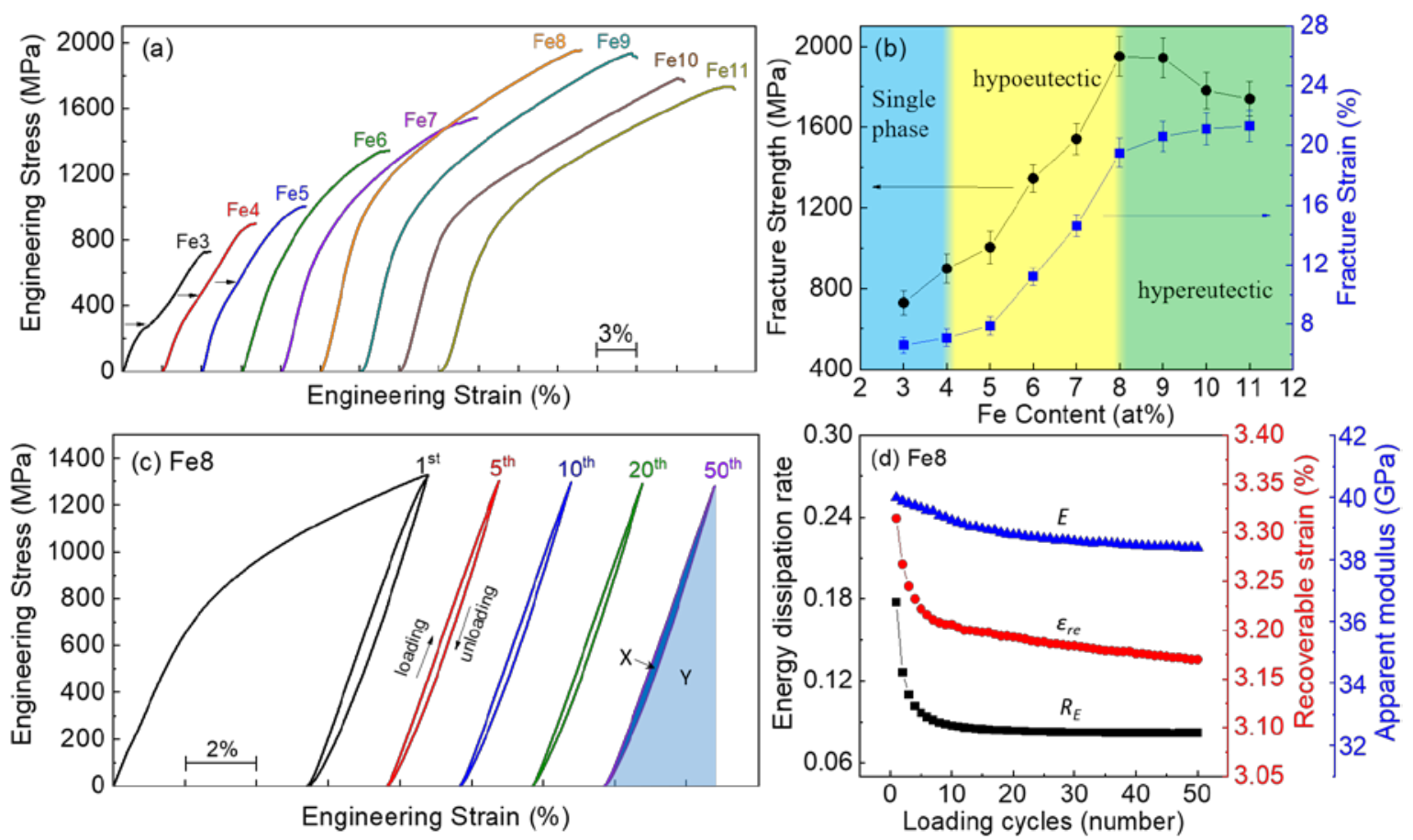

Fig. 6. Mechanical behaviour of $\mathrm{Ni}_{50} \mathrm{Mn}_{40-\mathrm{x}} \mathrm{Sn}_{10} \mathrm{Fe}_{\mathrm{x}}$ alloys. (a) Compressive engineering stress-strain curves of Fe3-Fe11 alloys, (b) Fracture strength and strain as functions of Fe content. (c) Compressive deformation cycling of Fe8. The stress-strain cycles were performed to the same total strain of $8.7 \%$. The selected stress-strain loops are displaced along the x-axis for a better presentation. (d) Evolutions of energy dissipation rate $\left(R_{E}\right)$, recoverable strain $\left(\varepsilon_{r e}\right)$ and the apparent Young's modulus $(E)$ during deformation cycling.

Fe8 was used for a cyclic compression test. Figure 6(c) shows a few selected loading-unloading cycles of the alloy, all deformed to $8.7 \%$ nominal strain. The first unloading showed a recovered strain of $3.3 \%$ and a residual strain of $5.4 \%$. With the subsequent loading-unloading cycles, the alloy exhibited quasilinear stress-strain loops with a small hysteresis $\left(\sim 1.5 \mathrm{~J} / \mathrm{cm}^{3}\right)$ after a few initial cycles. An energy dissipation ratio $\left(R_{E}\right)$ is defined as the area enclosed in a hysteresis loop (X) divided by the work input for the loading $(\mathrm{Y})$, as indicated on the $50^{\text {th }}$ cycle loop in the figure. The energy dissipation ratio $R_{E}$, the recoverable stain $(\varepsilon r e)$ and the apparent Young's modulus $(E)$ of the sample are presented in Figure $6(d)$ as functions of deformation cycles. It is seen that $R_{E}$ decreased rapidly in the initial five cycles and stabilised at approximately 0.08 with further cycling. The $\varepsilon$ re decreased slightly from $3.31 \%$ to $3.17 \%$ with deformation cycling. The apparent Young's modulus $E$ was measured to be below $40 \mathrm{GPa}$, with a slight decrease from 39.9 GPa to $38.4 \mathrm{GPa}$ following 50 deformation cycles. Such a low Young's modulus (thus high recoverable strain) is uncharacteristic of the elasticity of metallic materials. This implies that stress-induced martensitic transformation (SIMT) may have occurred in the deformation process, i.e. it is a "pseudoelastic" behaviour.

\subsection{Synchrotron HE-XRD characterisation}

To verify the pseudoelasticity of the deformation process, in-situ HE-XRD measurement was conducted during compressive deformation of Fe8. 
Figure 7(a) shows XRD diffraction spectrums of the composite when undeformed and deformed to $8.7 \%$. The $1 \mathrm{D}$ XRD spectrums were obtained by integrating the 2D diffraction patterns along all the azimuthal angles. Prior to compression, the alloy showed evident austenite peaks (e.g. (422)L2 $2_{1}$ with $d$-spacing of $1.222 \AA$ ), $\gamma$ phase peaks (e.g. (311) $\gamma$ with $d$ spacing of $1.097 \AA$ ) and a very weak martensite peak ((042) 4 o with $d$-spacing of $1.174 \AA)$. At deformation of $8.7 \%$, all the austenite peaks were reduced in intensity, while extra martensite peaks emerged, with all of them showing clear peak broadening. The presence of remaining austenitic peaks suggests the transformation was incomplete when compressed to $8.7 \%$. The $\gamma$ phase peaks all remained, but also showed line broadening with intensity reduction, except that for $(200)_{\gamma}$ peak at $1.819 \AA$ which shares an overlapping peak position with the martensite plane (321)40. The peak broadening of austenite, martensite and $\gamma$ phase is associated with the strain inhomogeneity with the increased defect density upon the plastic deformation of these phases. The above observation shows clear evidence for SIMT in the Heusler phase.
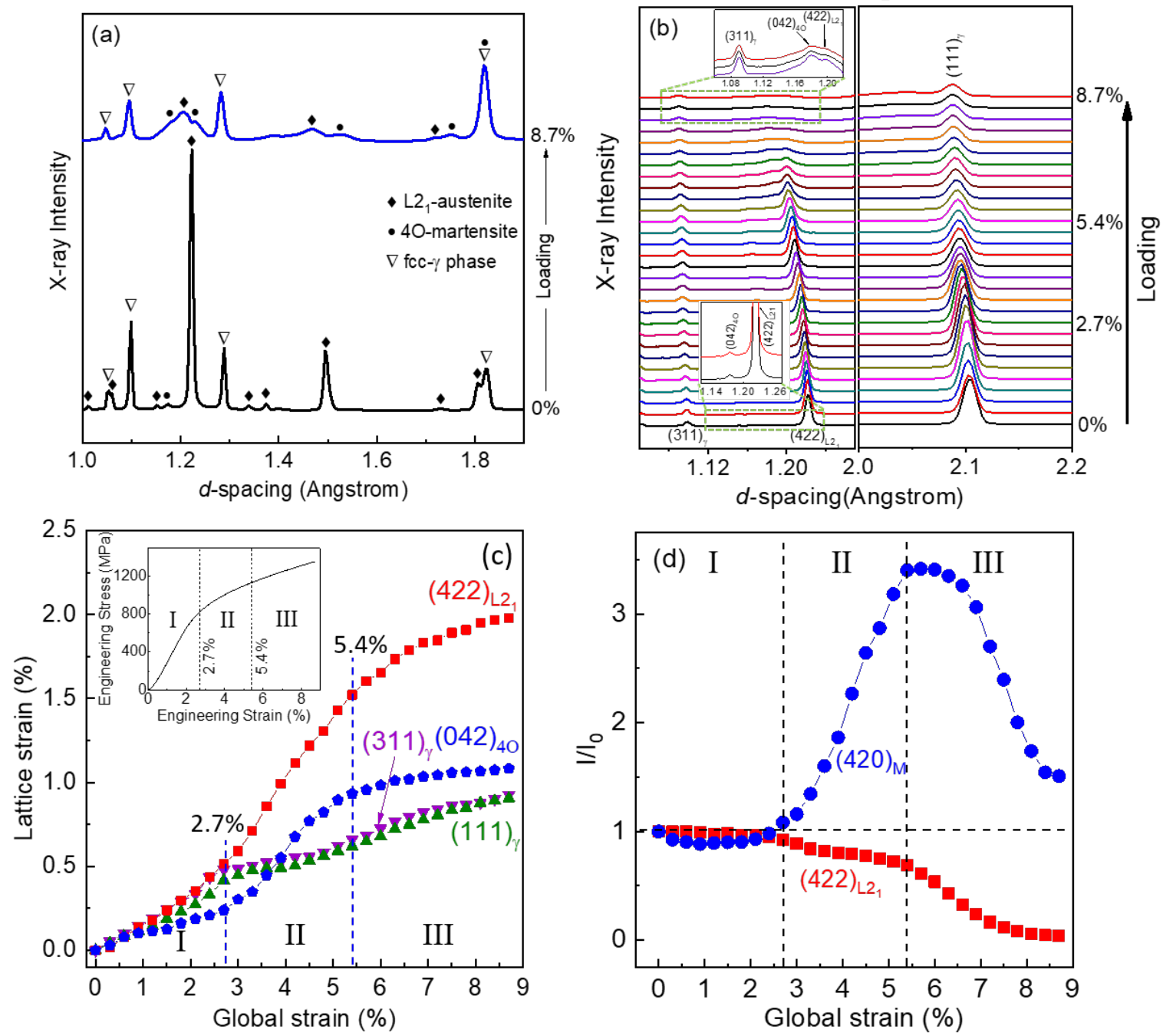

Fig. 7. Deformation behaviour of the Fe8 alloy. (a) 1D HE-XRD spectrums of the sample at $0 \%$ and $8.7 \%$ applied strains. The spectrums were $0-360^{\circ}$ integrated over the azimuth angle. (b) $1 \mathrm{D}$ HE-XRD spectrums along the loading direction collected at various stages during the compression. The bottom inset in (b) shows a local view at an enlarged Y scale to reveal the presence of the (042)40 peak prior to loading. The upper inset in (b) shows a local view at an enlarged Y scale at the end of loading, revealing (311) $\gamma$, (042) $4 \mathrm{O}$ and (422) $\mathrm{L}_{2}$ peaks. (c) 
Evolutions of $d$-spacing strains for $(422)_{\mathrm{L}_{2} 1},(042)_{40}$, (311) $\gamma$ and (111) $\gamma$ planes perpendicular to the loading direction during the compression. The inset in (c) shows the engineering compression stress-strain curve of the composite at room temperature. (d) Relative peak height intensity I/I0 of (420) M $_{\mathrm{M}}$ and (422) L2 $_{1}$ planes as functions of the global strain, where I is the peak height intensity at different global strains and $\mathrm{I}_{0}$ is the peak height intensity at zero applied stress.

Figure 7(b) shows 1D XRD diffraction spectrums of the composite in the loading direction during deformation to $8.7 \%$. It is evident that all diffraction peaks in the loading direction shifted to lower d-spacing values during loading, demonstrating the compressive elastic deformation in the axial direction. The lower inset in (b) shows the presence of a small martensite (042) 40 peak in the enlarged Y scale in the undeformed sample. The upper inset in (b) shows an enlarged Y scale and it shows that the (042)40 peak became stronger than the (422) L2 $_{1}$ peak with the progression of SIMT.

Figure 7(c) shows the lattice $d$-spacing strains of (422) $\mathrm{L} 21_{1},(042)_{40},(311)_{\gamma}$ and (111) $)_{\gamma}$ planes in the loading direction as functions of the applied strain. The lattice strain is calculated from the diffraction peak positions using $\varepsilon_{h k l}=\left|d_{h k l}-d_{h k l}^{0}\right| / d_{h k l}^{0}$, where $d_{h k l}^{0}$ is the peak position at zero applied stress. It is seen that the lattice strains evolved unevenly during the process of deformation, both within one itself and among them. Based on the behaviour of the various lattice strains, the deformation process is divided into three stages, as indicated in the figure.

In stage I, upon loading to $2.7 \%$ global strain, the lattice strains of (422) $\mathrm{L} 21_{1},(311)_{\gamma}$ and (111) $\gamma$ increased almost linearly. Given the very low apparent elastic modulus ( $<40 \mathrm{GPa}$, Figure 6(d)), this stage is apparently a mixed process of elastic deformation, some minor martensite reorientation, and possible stress-induced phase transformation. The lattice strain of (042)40 showed a smaller increase rate with the applied strain compared to those of (422)L2, (311) $\gamma$ and (111) $\gamma$ due to the reorientation of the pre-existing $4 \mathrm{O}$ martensite variants.

In stage II, from $2.7 \%$ to $5.4 \%$, the lattice strains of (311) $\gamma$ and (111) $\gamma$ increased at obviously reduced rates, while those of (422) $\mathrm{L}_{2}$ and (042) $4 \mathrm{O}$ increased more rapidly. The reduced rate of lattice strain increase of the $\gamma$ phase indicate its plastic yielding, which also coincides with yielding on the stress-strain curve shown in the inset in (c). This caused a load transfer from the $\gamma$ phase to the untransformed L2 ${ }_{1}$ austenite and the pre-existing 40 martensite, thus the increased rates of increase of their lattice strains ((422) $\mathrm{L} 2_{1}$ and (042) $4 \mathrm{O}$, respectively). In composites, load transfer occurs when one of the phases yields prior to others [52, 53]. In this case, the $\gamma$ phase yields first.

In stage III at above 5.4\% applied strain, the lattice strain of (422) $\mathrm{L}_{2}$ and (042) $4 \mathrm{O}$ continued to increase but at reduced rates, while the lattice strains of (311) $\gamma$ and (111) increased with slightly increased rates. This implies that the martensite and untransformed austenite had reached their elastic limits and started deforming plastically in this stage. The $\gamma$ phase continued to deform in a mixed mode of elastic and plastic deformations. 
The relative peak height intensity, defined as $\mathrm{I} / \mathrm{I}_{0}$, where $\mathrm{I}_{0}$ and $\mathrm{I}$ are the peak height intensities at zero load and different loads, is determined for the diffraction peaks in Figure 7(c). Figure 7 (d) shows the $\mathrm{I} / \mathrm{I}_{0}$ of (422) $\mathrm{L} 2_{2}$ and (042) 40 planes as functions of the applied strain in the longitudinal direction. In stage I, the I/I0 of (042) 40 showed a small decrease, which is due to the elastic deformation and reorientation of the pre-existing $4 \mathrm{O}$ martensite variants at this stage. The change of $\mathrm{I} / \mathrm{I}_{0}$ of (422) $\mathrm{L}_{2}$ is also very small, due to its elastic deformation. In stage II, the $\mathrm{I} / \mathrm{I}_{0}$ of (042) 40 exhibited a sharp rise while that of (422)L2 showed a moderate decrease. This evidences the progression of SIMT with the growing fraction of the $4 \mathrm{O}$ martensite at the expense of the L 21 austenite in the composite. The SIMT may be triggered by the load transfer from the $\gamma$ phase when its yielding point was reached at 2.7\% (Figure 6(c)). At above $5.4 \%$ of the applied strain (stage III), the I/I0 of (042) 4 o started to decline slowly first, then more rapidly. This implies massive plastic deformation emerged at around 5.4\% and became more dominantly with further deformation, which caused the broadening and reduced intensity of the martensite peaks. The $\mathrm{I} / \mathrm{I}_{0}$ of (422) $\mathrm{L}_{2}$ showed a greater rate of decrease compared to that in Stage II, which is apparently due to the combined effects by continued decrease in fraction along with the SIMT and the peak broadening by the massive plastic deformation upon further loading.

It is also noted that at the end of the loading process to $8.7 \%$ applied stain, the (422)L21, $(042)_{40}$, (311) $)_{\gamma}$ and (111) $)_{\gamma} d$-spacing strains reached $1.95 \%, 1.09 \%, 0.91 \%$ and $0.91 \%$, respectively. The $1.95 \%$ elastic strain of the $\mathrm{L} 21$ austenite and $1.09 \%$ of the $4 \mathrm{O}$ martensite are considered reasonable as the dislocation movement is difficult in their highly ordered structures. The maximum elastic strain of $0.91 \%$ of the $\gamma$ phase is unusual and significant. Yang et al. prepared a $\mathrm{Ni}_{59.1} \mathrm{Mn}_{13.1} \mathrm{Fe}_{15.2} \mathrm{Ga}_{12.6}$ alloy, which has a similar composition and structure to the $\gamma$ phase of Fe8, and measured a yield strength of $\sim 170 \mathrm{MPa}$ and a Young's modulus of $~ 80 \mathrm{GPa}$ [27]. Assuming the same Young's modulus for the $\gamma$ phase in Fe8, this lattice strain implies a stress of $728 \mathrm{MPa}$ at an applied strain of 8.7\%. The high strength and large elastic strain of the $\gamma$ phase are attributed to its reduced grain size.

\subsection{Fracture mechanisms of different microstructures}

Figure 8 shows the fractographic examination of Fe4, Fe6, Fe8 and Fe10 after deformation to rupture, as representatives of four distinctive microstructures. The single phase Fe4 alloy showed prevalent intergranular cracking along the original austenitic grain boundaries as shown in Figure 8(a). Some martensite lath can be seen on the grain surface. Some of the intergranular cracks are the result of the volume contraction of the martensite formation, and have contributed to its low strength and ductility. 

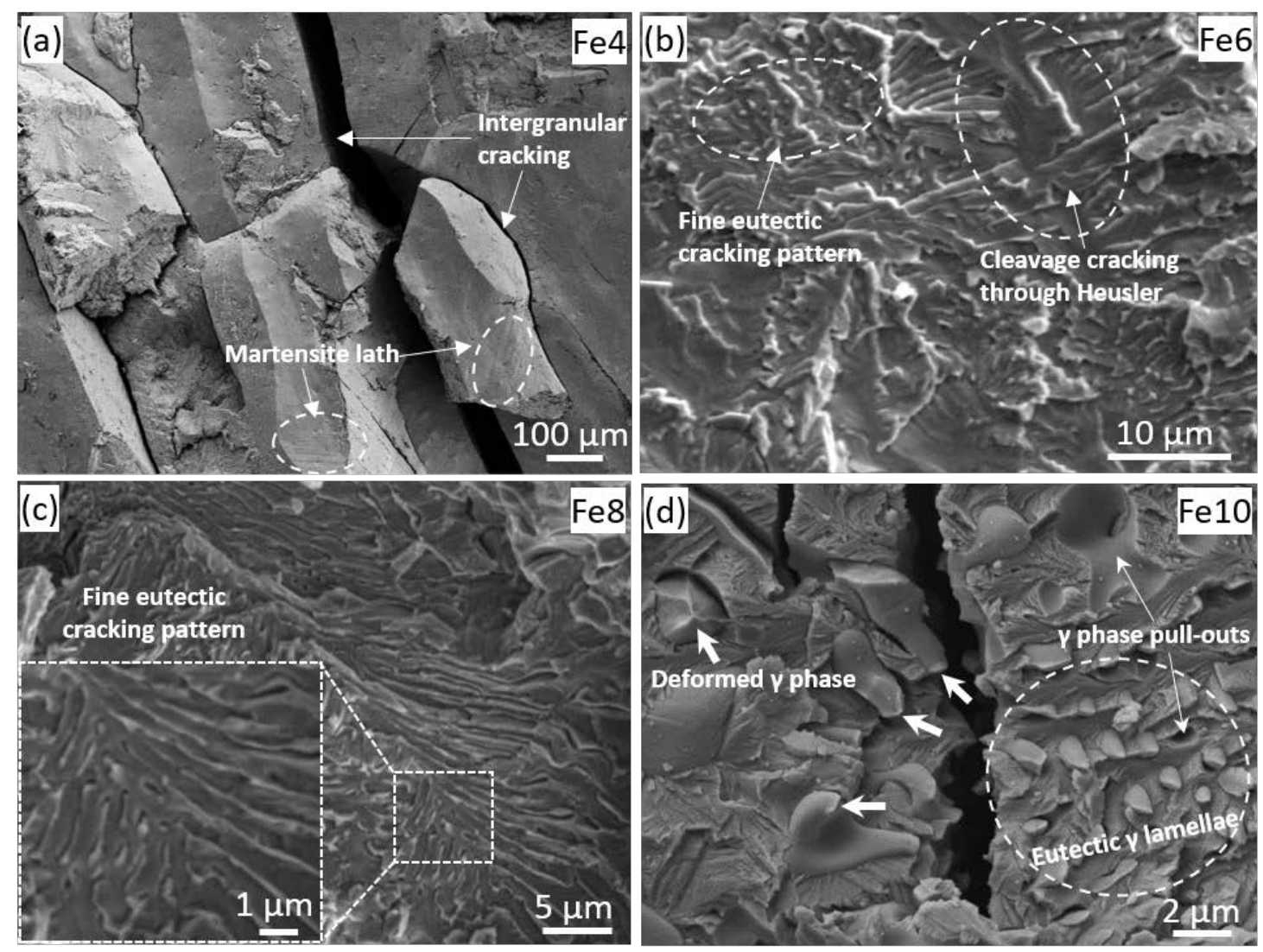

Fig. 8. Secondary SEM micrographs of the fracture surfaces of four different microstructure types: (a) Fe4 (single Heusler phase), (b) Fe6 (hypoeutectic), (c) Fe8 (eutectic) and (d) Fe10 (hypereutectic).

Figure 8(b) shows the fracture surface of the hypoeutectic Fe6 alloy. It exhibited a combination of the cleavage cracking of the pre-eutectic Heusler phase and fine cracking patterns of the eutectic lamellae. In this microstructure, cleavage cracks in the pre-eutectic Heusler phase are restricted by the $\gamma$ lamellae network, thus increasing the energy required for crack propagation. The effectiveness of retardation of crack propagation increases with increasing the volume fraction of the eutectic component. The eutectic Fe8 alloy showed a predominant fine eutectic cracking pattern on the fracture surface, as shown in Figure 8(c). The inset in (c) gives an enlarged view of the cracking surface, revealing the plastic stretch of the finely spaced $\gamma$ lamellae.

Figure 8(d) shows the fractured surface of Fe10. It reveals a combination of different cracking mechanisms. The circled region shows an array of the eutectic $\gamma$ phase lamellae embedded on a cleavage cracking face suggestive of their hindering effect on the crack extension. The tearing edges (thick arrows) of larger pre-eutectic $\gamma$ grains were also present, which suggest that they may have imposed a bridging effect on the cracks by plastic deformation and thus have contributed to ductility of the composite. The thin arrows indicate areas where the alloy experienced $\gamma$ phase pull-outs, indicative of the intergranular cracking along the interface [54]. The larger size and higher fraction of the $\gamma$ phase naturally promotes easier dislocation movement, but weakens the strain hardening effect (Figure 6(a)). 


\subsection{Thermomagnetic behaviours and magnetocaloric effect}

It is seen in Table 1 that addition of Fe and formation of the $\gamma$ phase change the chemical composition of the Heusler phase, in particularly the Mn and Sn contents. This will affect the martensitic transformation temperature, which may jeopardise the metamagnetic transformation and compromise the magnetic properties of the Heusler alloys. To clarify this, magnetisation (M-T) measurements under a small magnetic field of 100 Oe were carried out in the temperature range of 10-350 K, as shown in Figure 9(a).

The single phase Fe3 and Fe4 alloys showed very low magnetisations at above $250 \mathrm{~K}$. The inset in (a) shows a local view of the M-T curves within 260 340 K on an enlarged Y scale, revealing a small magnetisation peak in each curve. This is the Curie transition $\left(T_{C}^{A}\right)$ of the remnant austenite in the predominantly martensite in the alloys. The immediate drop of the magnetisation indicates the transformation of the remnant austenite to a paramagnetic martensite. At below $250 \mathrm{~K}$, the alloys showed a gradual increase of magnetisation due to the Curie transition of the martensite $\left(T_{C}^{M}\right)$.

The hypoeutectic Fe5, Fe6 and Fe7 alloys showed a similar M-T behaviour. Upon cooling, the magnetisation of the alloys increased sharply at around $320 \mathrm{~K}$, and then decreased to reach a minimum at around $260 \mathrm{~K}$ before increased again on further cooling. The first rapid increase of magnetisation corresponds to the Curie transition $\left(T_{C}^{A}\right)$ of the $\mathrm{L} 2{ }_{1}$ austenite in the alloys. The immediate decrease after $T_{C}^{A}$ indicates the transformation from the ferromagnetic L 21 austenite to the paramagnetic $4 \mathrm{O}$ martensite of the pre-eutectic Heusler phase (i.e. the metamagnetic transformation). The second gradual increase in magnetisation is due to the Curie transition of the paramagnetic 40 martensite $\left(T_{C}^{M}\right)$ of the pre-eutectic Heusler phase. The magnetisation decreased again upon cooling to below $200 \mathrm{~K}$, and the magnitude of the decrease is greater in alloys with higher Fe contents, i.e. with higher fractions of the eutectic Heusler phase. Therefore, this decrease is attributed to the transformation of the ferromagnetic austenite in the eutectic to the weak magnetic martensite. Due to the nature of dendritic solidification (Figure 1(c)), there were composition gradients within the Heusler dendrites, which results in the continuous transformation over a wide temperature span (100-300 K).

Fe8 showed a single-step martensitic transformation at a lower transformation temperature. The transformation temperatures are $\mathrm{M}_{\mathrm{s}}=110 \mathrm{~K}, \mathrm{M}_{\mathrm{f}}=81 \mathrm{~K}, \mathrm{~A}_{\mathrm{s}}=109 \mathrm{~K}, \mathrm{~A}_{\mathrm{f}}=139 \mathrm{~K}$ and the transformation hysteresis is approximately $29 \mathrm{~K}$. Fe9 showed the sign of the martensitic transformation at below $100 \mathrm{~K}$, but the transformation seems to be incomplete within the temperature range measured. The M-T curves of Fe10 and Fe11 did not show any sign of martensitic transformation. The lowered transformation temperatures are due to the reduced e/a ratios of the Heusler phase in the alloys with higher Fe contents (Table 1). These observations demonstrated that the $\mathrm{L} 21 \rightarrow 4 \mathrm{O}$ martensitic transformation occurs at e/a $>8.041$. 

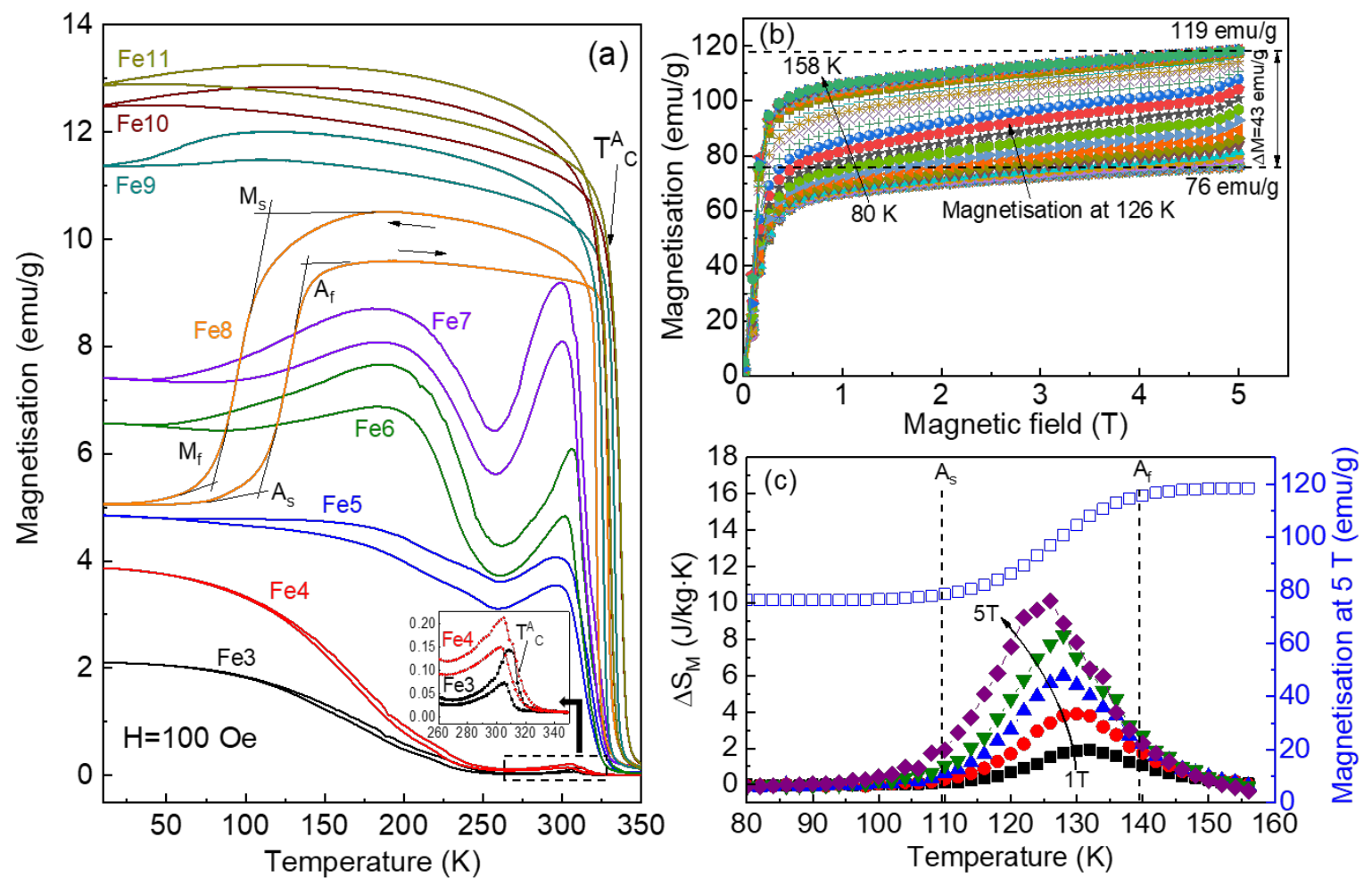

Fig. 9. Thermomagnetic properties of the $\mathrm{Ni}_{50} \mathrm{Mn}_{40-\mathrm{x}} \mathrm{Sn}_{10} \mathrm{Fe}_{\mathrm{x}}$ alloys. (a) M-T curves of Fe3Fe11 under a small magnetic field of 100 Oe. The inset shows an enlarged view of the M-T curves of $\mathrm{Fe} 3$ and $\mathrm{Fe} 4$ at between $260 \mathrm{~K}$ and $350 \mathrm{~K}$ (dashed box in (a)). (b) M-H isothermal curves measured at different temperatures of Fe8. (c) The magnetic entropy change $\Delta S_{M}$ associated with the reverse $4 \mathrm{O} \rightarrow \mathrm{L} 21$ transformation under different fields of 1-5 $\mathrm{T}$ and maximum magnetisations at $5 \mathrm{~T}$ as functions of temperature of Fe8.

Figure 9(b) shows the isothermal magnetisation behaviour of Fe8 measured at different temperatures from $80 \mathrm{~K}$ to $158 \mathrm{~K}$ covering the reverse transformation. It is seen that both the $4 \mathrm{O}$ martensite (at $80 \mathrm{~K}$ ) and L21 austenite (at $158 \mathrm{~K}$ ) exhibited a spontaneous magnetisation, indicative of their ferromagnetic state. However, the maximum magnetisation of the two phases at $5 \mathrm{~T}$ are different, with that of the $4 \mathrm{O}$ martensite being $76 \mathrm{emu}$ at $80 \mathrm{~K}$ and that of the $\mathrm{L} 21$ austenite being $119 \mathrm{emu} / \mathrm{g}$ at $158 \mathrm{~K}$. At temperatures between $\mathrm{A}_{\mathrm{s}}$ and $\mathrm{A}_{\mathrm{f}}$, the alloy showed the maximum magnetisations between 76 and $119 \mathrm{emu} / \mathrm{g}$ at $5 \mathrm{~T}$, indicating a mixed state of the austenite and martensite, or the progress of the reverse $4 \mathrm{O} \rightarrow \mathrm{L} 22_{1}$ transformation.

The magnetic entropy change $\left(\Delta \mathrm{S}_{\mathrm{M}}\right)$ of the reverse transformation can be estimated from the $\mathrm{M}-\mathrm{H}$ measurements shown in Figure 9(b) using the Maxwell equation [55]. The estimated $\Delta \mathrm{S}_{M}$ under different field strengths are plotted in Figure 9(c) as functions of temperature of Fe8. Also plotted in the figure are the maximum magnetisation at $5 \mathrm{~T}$ of the sample at different temperatures. It is seen that the reverse transformation occurred from $109 \mathrm{~K}$ to $139 \mathrm{~K}$, consistent with the M-T measurement shown in (a). The maximum $\Delta \mathrm{S}_{\mathrm{M}}$ achieved at $5 \mathrm{~T}$ is 10.2 $\mathrm{J} / \mathrm{kg} \cdot \mathrm{K}$. Considering that the eutectic Fe8 contained $30.4 \% \gamma$ phase (assuming same specific density for the Heusler phase and the $\gamma$ phase), this corresponds to $14.7 \mathrm{~J} / \mathrm{kg} \cdot \mathrm{K}$ for the Heusler phase in the alloy. This value is still smaller than those reported in the single phase Heusler 
alloys, such as $\Delta \mathrm{S}_{\mathrm{M}}=32 \mathrm{~J} / \mathrm{kg} \cdot \mathrm{K}$ at $5 \mathrm{~T}$ in a $\mathrm{Ni}_{48} \mathrm{Co}_{2} \mathrm{Mn}_{38} \mathrm{Sn}_{12}$ alloy [56] and $\Delta \mathrm{S}_{\mathrm{M}}=22 \mathrm{~J} / \mathrm{kg} \cdot \mathrm{K}$ at $2 \mathrm{~T}$ in a Ni50.2 $\mathrm{Mn}_{35.0} \mathrm{In}_{14.8}$ alloy [57]. The refrigerant capacity, defined as $\mathrm{RC}=\int_{\mathrm{T}_{1}}^{\mathrm{T}_{2}}\left|\Delta \mathrm{S}_{\mathrm{M}}\right| \mathrm{dT}$, where $T_{1}$ and $T_{2}$ are the temperatures for the hot and cold sinks in one refrigeration cycle, is an important figure-of-merit for refrigeration application [58]. Integrating the $\Delta \mathrm{S}_{\mathrm{M}}$ curve at $5 \mathrm{~T}$ over the transformation temperatures range $\left(\mathrm{T}_{1}=\mathrm{A}_{\mathrm{s}}\right.$ and $\mathrm{T}_{2}=\mathrm{A}_{\mathrm{f}}$ ), the $\mathrm{RC}$ value is determined to be $181 \mathrm{~J} / \mathrm{kg}$, or $260 \mathrm{~J} / \mathrm{kg}$ for the Heusler phase only. This value is greater than the highest reported RC value of Heusler alloys being $168 \mathrm{~J} / \mathrm{kg}$ under the same condition in a $\mathrm{Ni}_{2} \mathrm{Mn}_{1.9} \mathrm{Cr}_{0.1}$ In alloy [59].

\section{Discussion}

Many attempts have been made to obtain various dual-phase microstructures with different amounts of $\gamma$ phase in Ni-(Co)-Mn-X (X=Ga, In, Sn and Sb) Heusler alloys to improve the mechanical performance of the alloys. The composition of the Heusler phase and its e/a ratio can also be significantly affected due to the formation of the $\gamma$ phase (Table 1), which in turn changes the martensitic transformation temperatures [49]. In the literature, the e/a ratio of the Heusler phase has often been found to increase with the $\gamma$ phase fraction and exhibit very high martensitic transformation temperatures above $T_{C}^{A}[17,22,24,26,29,60]$. These alloys are potential candidates for high temperature shape memory alloys. However, it also implies the decoupling of the magnetic transition from the martensitic transformation $\left(T_{C}^{A}>M_{S}\right)$, thus losing the characteristics of the metamagnetic phase transformation in these alloys.

Figure 10 summarises the compressive strength and ductility of the reported ferromagnetic dual-phase Heusler alloys which satisfy the condition of $T_{C}^{A}>M_{s}$. It is evident that our Fe8 and $\mathrm{Fe} 9$ alloys outperform the rest in both strength and ductility. The superior mechanical properties of Fe8 and Fe9 are attributed to their refined microstructures in the eutectic.

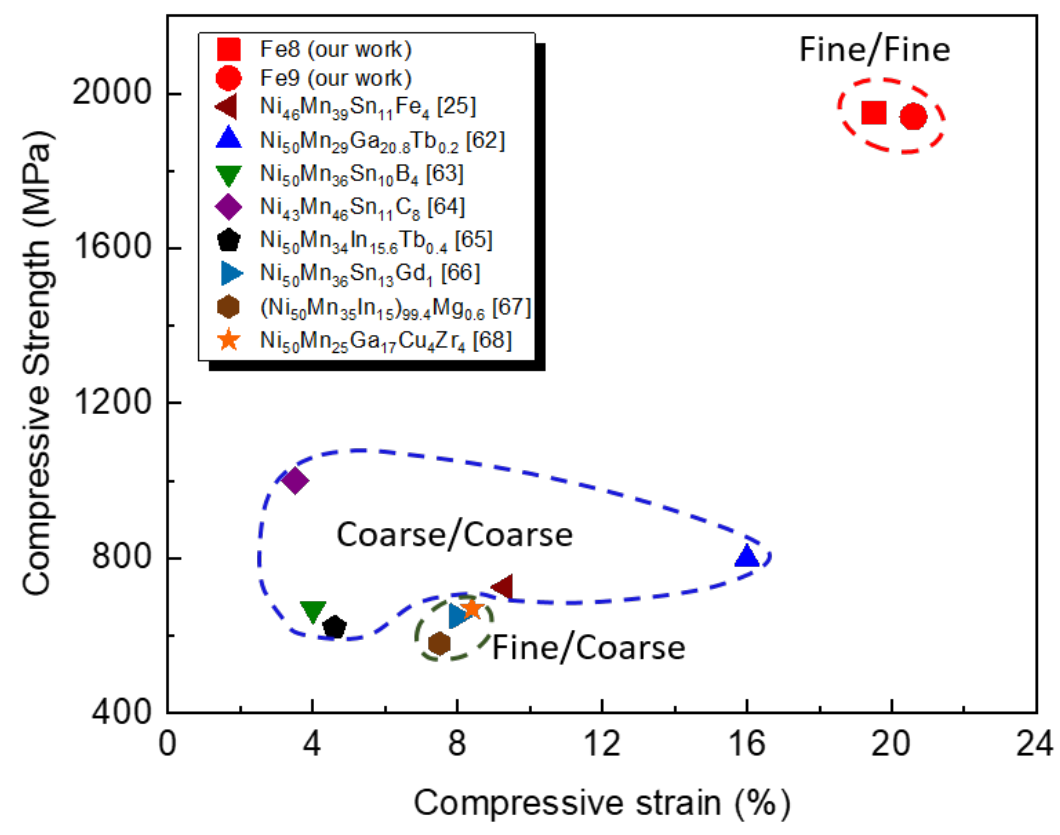

Fig. 10. Comparison of the mechanical properties of Fe8 and Fe9 alloys with those of other dualphase Heusler alloys with a condition of $T_{C}^{A}>M_{S}$. 
The toughening strategy for Heusler alloys is to hinder crack propagation in the brittle Heusler phase by the introduction of the ductile $\gamma$ phase. The optimisation of this strategy can be achieved by maximising the departmentalisation of the brittle Heusler matrix using $\gamma$ phase with suitable grain size, and such strategy is analogous to the departmentalisation of a dislocation slip matrix for achieving high toughness and large strains of nanoinclusions in a $\mathrm{NiTi} / \mathrm{Ti}_{3} \mathrm{Sn}$ system [61]. For a dual-phase structure, four possible configurations may be envisaged based on the dimension and interspacing of the $\gamma$ phase, which are schematically shown in Figure 11. For simplicity, an ellipsoidal shape of two different sizes are used to represent the coarse and fine $\gamma$ phase grains and the hexagons represent the Heusler matrix with a typical equiaxed grain structure.

Figure 11(a) shows a configuration of coarse $\gamma$ phase grains embedded in Heusler matrix with coarse spacing, designated as the Coarse/Coarse configuration. The dual-phase morphology of alloys reported in Refs [25], [62], [63], [64] and [65], which are shown in Figure 10, belongs to this configuration. Obviously, intergranular and transgranular cracking can readily propagate through the large spacing among the $\gamma$ phase grains. Figure 11(b) shows a Fine/Coarse configuration, which can be observed in alloys reported in Refs [66], [67] and [68]. Similar to the Coarse/Coarse configuration, the impedance effect on crack propagation by the $\gamma$ phase grains is also very limited in this configuration due to their large interspacing.

Figure 11(c) shows a Coarse/Fine configuration, in which the crack has an impaired path through the brittle Heusler matrix. Under load, crack bridging can form by plastic stretching of the ductile $\gamma$ phase grains thus increasing the resistance to crack propagation and the ductility of the composite [26,69]. It is apparent that this morphology requires high fraction of the $\gamma$ phase, which will inevitably reduce the efficiency of functionalities associated with the martensitic transformation of the alloy, such as magnetocaloric and magnetoresistance effects. A high volume fraction of the $\gamma$ phase also normally results in large changes of the martensitic transformation temperature. In fact, none of the reported Heusler composites with a Coarse/Coarse configuration can fulfil the $T_{C}^{A}>M_{S}$ condition due to the difficult control of the composition of the Heusler phase. In addition, the excessive plastic deformation in coarse grained $\gamma$ phase will also severely impede the shape memory effect and superelasticity of the Heusler phase. This is another drawback of this morphology. 


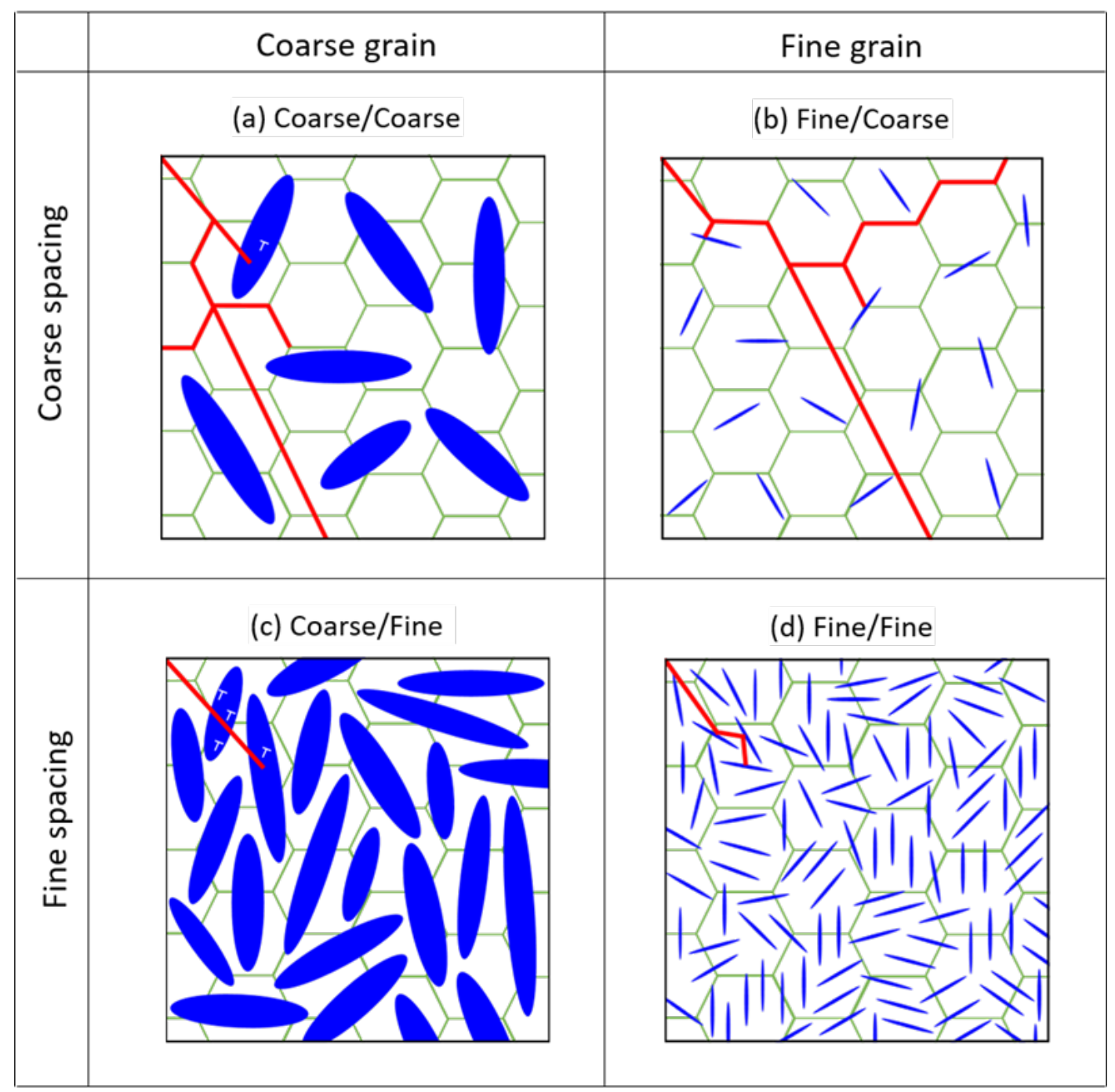

Fig. 11. Classification schema of departmentalisation configurations for Heusler- $\gamma$ phase composite according to the dimension and interspacing of the $\gamma$ phase: (a) the Coarse/Coarse configuration: coarse grain with coarse spacing, (b) the Fine/Coarse configuration: fine grain with coarse spacing, (c) the Coarse/Fine configuration: coarse grain with fine spacing and (d) the Fine/Fine configuration: fine grain with fine spacing. Morphology (c) has a high volume fraction, morphologies (a) and (d) have medium volume fractions, and morphology (b) has a small volume fraction of the $\gamma$ phase.

Figure 11(d) shows a Fine/Fine configuration. Passage of cracks through the Heusler matrix is significantly restricted by the finely interspaced $\gamma$ phase grains. Upon deformation, in addition to the crack bridging effect, the fine $\gamma$ phase grains (at sub-micron scale) are also more resistant to plastic deformation than the coarse ones due to the relative starvation of slip systems, i.e. the "smaller is stronger" principle [70-72]. This may force the crack to propagate (or deflect) between the small $\gamma$ phase grains, known as the crack trapping effect [73, 74]. The associated perturbation of the crack front requires an increase in the required stress intensity factor for this propagation, hence an increased fracture toughness. The crack trapping may be more predominant with decreasing the grain size of the $\gamma$ phase. More importantly, the benefit of fine sized $\gamma$ phase is the capability of large elastic strain, which can better match with the lattice distortion strain of a SIMT process at the dual-phase interface. It is noted that the average $\gamma$ 
phase lamellae thickness in Fe8 reached down to $170 \mathrm{~nm}$. Further grain refinement is desirable and may be obtained by increasing the solidification cooling rate.

The effectiveness of crack restriction, elastic strain capability and volume fraction of the four configurations are summarised in Table 2. The Fine/Fine configuration is more preferable than the other three configurations, due to its great crack restriction capability, large elastic strain and reasonable volume fraction in the composite.

Table 2. Comparison of crack restriction, elastic strain capability and volume fraction among different departmentalisation configurations of the $\gamma$ phase in a Heusler matrix.

\begin{tabular}{|c|c|c|c|c|}
\hline & \multicolumn{2}{|c|}{ Coarse grain } & \multicolumn{2}{c|}{ Fine grain } \\
\cline { 2 - 5 } & $\begin{array}{c}\text { Coarse spacing } \\
\text { (Coarse/Coarse) }\end{array}$ & $\begin{array}{c}\text { Fine spacing } \\
\text { (Coarse/Fine) }\end{array}$ & $\begin{array}{c}\text { Coarse spacing } \\
\text { (Fine/Coarse) }\end{array}$ & $\begin{array}{c}\text { Fine spacing } \\
\text { (Fine/Fine) }\end{array}$ \\
\hline $\begin{array}{c}\text { Crack } \\
\text { Restriction }\end{array}$ & $x$ & $\checkmark$ & $x$ & $\checkmark$ \\
\hline $\begin{array}{c}\text { Large elastic } \\
\text { strain }\end{array}$ & $x$ & $x$ & $\checkmark$ & $\checkmark$ \\
\hline $\begin{array}{c}\text { Volume fraction } \\
\text { of } \gamma \text { phase }\end{array}$ & Medium & High & Low & Medium \\
\hline
\end{tabular}

\section{Conclusions}

This study investigated a new strategy for toughening of dual-phase Heusler alloys by microstructural refinement via eutectic solidification, as demonstrated in Ni50Mn40-x $\mathrm{Sn}_{10} \mathrm{Fe}_{\mathrm{x}}$ alloys. The eutectic microstructure exhibited superior mechanical properties while retaining the metamagnetic phase transformation. The main conclusions can be drawn as follows:

1) The alloys showed a microstructure evolution from single Heusler phase $\rightarrow$ hypoeutectic $\rightarrow$ Heusler- $\gamma$ eutectic $\rightarrow$ hypereutectic microstructures with increasing the Fe addition. Formation of the $\gamma$ phase modified the composition of the Heusler matrix. Both the e/a ratio and the $\mathrm{L} 21 \rightarrow 4 \mathrm{O}$ martensitic transformation temperature decreased with increasing the Fe addition.

2) The best mechanical properties were found in the alloys with nearly fully eutectic microstructures with fine $\gamma$ phase thickness and spacing. The highest compressive strength achieved was $1950 \mathrm{MPa}$ in Fe8, which also had a ductility of 19.5\%. Such dual-phase morphology also showed stable mechanical cyclability, a low apparent Young's Modulus of $\sim 38 \mathrm{GPa}$ and large superelasticity of $\sim 3.2 \%$.

3) A typical metamagnetic phase transformation was achieved in Fe8. A moderate $\Delta \mathrm{S}_{\mathrm{M}}$ of $10.2 \mathrm{~J} / \mathrm{kg} \cdot \mathrm{K}$ and a large refrigerant capacity of $181 \mathrm{~J} / \mathrm{kg}$ with a magnetic field change of $5 \mathrm{~T}$ were obtained in this alloy. These values are comparable to those reported for single phase NiMn-based Heusler alloys.

\section{Acknowledgements}

The authors wish to acknowledge the financial supports from the Natural Science Foundation of Guangdong Province (Grant No.:2018A030313742), Australian Research Council (Grant 
No.: DP180101955, DP190102990 and DE150101795) and National Natural Science Foundation of China (Grant No.: 51671024). We acknowledge the facilities, and the scientific and technical assistance of the Australian National Fabrication Facility at the Centre for Microscopy, Characterisation \& Analysis, The University of Western Australia, a facility funded by the University, State and Commonwealth Governments. This research used resources of the Advanced Photon Source, a U.S. Department of Energy (DOE) Office of Science User Facility operated for the DOE Office of Science by Argonne National Laboratory under Contract No. DE-AC02-06CH11357.

\section{Reference}

[1] K. Ullakko, Magnetically Controlled Shape Memory Alloys: A New Class of Actuator Materials Journal of Materials Engineering and Performance 5(3) (1996) 405-409.

[2] A. Hubert, N. Calchand, Y. Le Gorrec, J.Y. Gauthier, Magnetic Shape Memory Alloys as Smart Materials for Micro-positioning Devices Advanced Electromagnetics 1(2) (2012) 1-10.

[3] N.G.S.H. Khan, Review of Properties of Magnetic Shape Memory (MSM) Alloys and MSM Actuator Designs Journal of Physics: Conference Series 588 (2015) 012052.

[4] O. Heczko, A. Sozinov, K. Ullakko, Giant field-induced reversible strain in magnetic shape memory NiMnGa alloy IEEE Transactions on Magnetics 36(5) (2000) 3266-3268.

[5] H.E. Karaca, I. Karaman, B. Basaran, Y.I. Chumlyakov, H.J. Maier, Magnetic field and stress induced martensite reorientation in NiMnGa ferromagnetic shape memor alloy single crystals, Acta Materialia 54(1) (2006) 233-245.

[6] Y. Ganor, D. Shilo, T.W. Shield, R.D. James, Breaching the work output limitation of ferromagnetic shape memory alloys, Applied Physics 93 (2008) 122509.

[7] R. Kainuma, Y. Imano, W. Ito, Y. Sutou, H. Morito, S. Okamoto, O. Kitakami, K. Oikawa, A. Fujita, T. Kanomata, K. Ishida, Magnetic-field-inudced shape recovery by reverse phase transformation, Nature 439 (2006) 957-960.

[8] R. Kainuma, Y. Imano, W. Ito, H. Morito, Y. Sutou, K. Oikawa, A. Fujita, K. Ishida, S. Okamoto, O. Kitakami, T. Kanomata, Metamagnetic shape memory effect in a Heusler-type Ni43Co7Mn39Sn11 polycrystalline alloy, Applied Physics Letters 88 (2006) 192513.

[9] J. Liu, T. Gottschall, K.P. Skokov, J.D. Moore, O. Gutfleisch, Giant magnetocaloric effect driven by structural transitions, Nature Materials 11(7) (2012) 620-626.

[10] K. Keiichi, O. Hironari, W. Kazuo, K. Takeshi, K. Ryosuke, I. Wataru, O. Katsunari, I. Kiyohito, Observation of large magnetoresistance of magnetic Heusler alloy Ni50Mn36Sn14 in high magnetic fields, Applied Physics Letters 89(18) (2006) 182510.

[11] B. Lu, F. Xiao, A. Yan, J. Liu, Elastocaloric effect in a textured polycrystalline Ni-MnIn-Co metamagnetic shape memory alloy, Applied Physics Letters 105(16) (2014) 161905.

[12] Z. Yang, D.Y. Cong, L. Huang, Z.H. Nie, X.M. Sun, Q.H. Zhang, Y.D. Wang, Large elastocaloric effect in a Ni-Co-Mn-Sn magnetic shape memory alloy, Materials \& Design 92 (2016) 932-936.

[13] Z. Wu, Z. Liu, H. Yang, Y. Liu, G. Wu, R.C. Woodward, Metallurgical origin of the effect of Fe doping on the martensitic and magnetic transformation behaviours of Ni50Mn40xSn10Fex magnetic shape memory alloys, Intermetallics 19(4) (2011) 445-452.

[14] L. Manosa, X. Moya, A. Planes, O. Gutfleisch, J. Lyubina, M. Barrio, J.-L. Tamarit, S. Aksoy, T. Krenke, M. Acet, Effects of hydrostatic pressure on the magnetism and martensitic 
transition of Ni-Mn-In magnetic superelastic alloys, Applied Physics Letters 92(1) (2008) 012515-3.

[15] X. He, S. Wei, Y. Kang, Y. Zhang, Y. Cao, K. Xu, Z. Li, C. Jing, Enhanced barocaloric effect produced by hydrostatic pressure-induced martensitic transformation for Ni44.6Co5.5Mn35.5In14.4 Heusler alloy, Scripta Materialia 145 (2018) 58-61.

[16] C.O. Aguilar-Ortiz, D. Soto-Parra, P. Álvarez-Alonso, P. Lázpita, D. Salazar, P.O. Castillo-Villa, H. Flores-Zúñiga, V.A. Chernenko, Influence of Fe doping and magnetic field on martensitic transition in Ni-Mn-Sn melt-spun ribbons, Acta Materialia 107 (2016) 9-16.

[17] Y. Ma, C. Jiang, Y. Li, H. Xu, C. Wang, X. Liu, Study of Ni50+xMn25Ga25-x ( $x=2-11)$ as high-temperature shape-memory alloys, Acta materialia 55(5) (2007) 1533-1541.

[18] Y. Feng, J. Sui, Z. Gao, W. Cai, Investigation on martensitic transformation and mechanical properties of the Ni50Mn37-xSn13Fex (x=0, 2, 5, 10) alloys, International Journal of Modern Physics B 23 (2010) 1803-1808.

[19] Y. Feng, J.H. Sui, H.B. Wang, W. Cai, Reversible magnetic-field-induced phase transformation and magnetocaloric effect above room temperature in a Ni-Mn-In-Fe Polycrystal, Journal of Magnetism and Magnetic Materials 324(12) (2012) 1982-1984.

[20] Y. Feng, J.H. Sui, Z.Y. Gao, G.F. Dong, W. Cai, Microstructure, phase transitions and mechanical properties of Ni50Mn34In16-yCoy alloys, Journal of Alloys and Compounds 476(1-2) (2009) 935-939.

[21] S. Yang, Y. Ma, H. Jiang, X. Liu, Microstructure and shape-memory characteristics of Ni $56 \mathrm{Mn} 25-\mathrm{x}$ Co x Ga 19 ( x =4, 8) high-temperature shape-memory alloys, Intermetallics 19(2) (2011) 225-228.

[22] Y. Ma, S. Lai, S. Yang, Y. Luo, C. Wang, X. Liu, Ni56Mn25-xCrxGa19 (x=0, 2, 4, 6) high temperature shape memory alloys, Transactions of Nonferrous Metals Society of China 21(1) (2011) 96-101.

[23] Y. Xin, Y. Zhou, Martensitic transformation and mechanical properties of NiMnGaV high-temperature shape memory alloys, Intermetallics 73 (2016) 50-57.

[24] Y. Ma, S. Yang, W. Jin, X. Liu, Ni56Mn25-xCuxGa19 ( $x=0$, 1, 2, 4, 8) high-temperature shape-memory alloys, Journal of Alloys and Compounds 471(1-2) (2009) 570-574.

[25] C. Tan, Z. Tai, K. Zhang, X. Tian, W. Cai, Simultaneous enhancement of magnetic and mechanical properties in Ni-Mn-Sn alloy by Fe doping, Scientific reports 7 (2017) 43387.

[26] Y. Ma, S. Yang, Y. Liu, X. Liu, The ductility and shape-memory properties of Ni-Mn-CoGa high-temperature shape-memory alloys, Acta Materialia 57(11) (2009) 3232-3241.

[27] S.Y. Yang, Y. Liu, C.P. Wang, Z. Shi, X.J. Liu, The mechanism clarification of Ni-Mn$\mathrm{Fe}-\mathrm{Ga}$ alloys with excellent and stable functional properties, Journal of Alloys and Compounds 560 (2013) 84-91.

[28] Y. Xu, B. Lu, W. Sun, A. Yan, J. Liu, Large and reversible elastocaloric effect in dualphase Ni54Fe19Ga27 superelastic alloys, Applied Physics Letters 106(20) (2015) 201903.

[29] Y. Feng, J.H. Sui, Z.Y. Gao, J. Zhang, W. Cai, Investigation on martensitic transformation behavior, microstructures and mechanical properties of Fe-doped Ni-Mn-In alloys, Materials Science and Engineering A 507(1-2) (2009) 174-178.

[30] S. Yang, C. Wang, Z. Shi, J. Wang, J. Zhang, Y. Huang, X. Liu, Microstructure, martensitic transformation, mechanical and shape memory properties of Ni-Co-Mn-In high- 
temperature shape memory alloys under different heat treatments, Materials Science and Engineering: A 655 (2016) 204-211.

[31] Y. Ma, S. Yang, Y. Liu, X. Liu, Tensile characteristics and shape memory effect of NiMnCoGa high-temperature shape memory alloy, Scripta Materialia 58 (2008) 918-921.

[32] Y. Ma, S. Yang, Y. Liu, X. Liu, NiMnCuGa $(x=0,1,2,4,8)$ high-temperature shapememory alloys, Journal of Alloys and Compounds 471 (2009) 570-574.

[33] S. Yang, Y. Liu, C. Wang, Y. Lu, J. Wang, Z. Shi, X. Liu, Microstructure and functional properties of two-phase $\mathrm{Ni}-\mathrm{Mn}-\mathrm{Fe}-\mathrm{In}$ shape memory alloys with small transformation hysteresis width, Journal of Alloys and Compounds 619 (2015) 498-504.

[34] J. Sui, X. Zhang, L. Gao, W. Cai, Microstructure, phase transformation and mechanical properties of Ni-Mn-Ga-Y magnetic shape memory alloys, Journal of Alloys and Compounds 509(35) (2011) 8692-8699.

[35] P.M. Anderson, J.P. Hirth, J. Lothe, Theory of dislocations, Cambridge University Press2017.

[36] K. Otsuka, C.M. Wayman, Shape memory materials, Cambridge university press1999.

[37] S. Hao, L. Cui, D. Jiang, X. Han, Y. Ren, J. Jiang, Y. Liu, Z. Liu, S. Mao, Y. Wang, Y. Li, X. Ren, X. Ding, S. Wang, C. Yu, X. Shi, M. Du, F. Yang, Y. Zheng, Z. Zhang, X. Li, D.E. Brown, J. Li, A Transforming Metal Nanocomposite with Large Elastic Strain, Low Modulus, and High Strength, Science 339(6124) (2013) 1191-1194.

[38] Z.W. Shan, R.K. Mishra, S.A. Syed Asif, O.L. Warren, A.M. Minor, Mechanical annealing and source-limited deformation in submicrometre-diameter Ni crystals, Nature Materials 7 (2007) 115.

[39] S. Suresh, J. Li, Materials science: Deformation of the ultra-strong, Nature 456(7223) (2008) 716.

[40] E. Wachtel, F. Henninger, B. Predel, Constitution and magnetic properties of Ni-Mn-Sn alloys - solid and liquid state, Journal of Magnetism and Magnetic Materials 38(3) (1983) 305315.

[41] L.J. Swartzendruber, V.P. Itkin, C.B. Alcock, The Fe-Ni (iron-nickel) system, Journal of Phase Equilibria 12(3) (1991) 288-312.

[42] V.T. Witusiewicz, F. Sommer, E.J. Mittemeijer, Reevaluation of the Fe-Mn phase diagram, Journal of Phase Equilibria and Diffusion 25(4) (2004) 346-354.

[43] N.A. Gokcen, The Mn-Ni (Manganese-Nickel) System, Journal of Phase Equilibria 12(3) (1991) 313-321.

[44] K.C.H. Kumar, P. Wollants, L. Delaey, Thermodynamic evaluation of Fe-Sn phase diagram, Calphad 20(2) (1996) 139-149.

[45] L. Caron, Z.Q. Ou, T.T. Nguyen, D.T. Cam Thanh, O. Tegus, E. Bruck, On the determination of the magnetic entropy change in materials with first-order transitions, Journal of Magnetism and Magnetic Materials 321(21) (2009) 3559-3566.

[46] M. Földeàki, R. Chahine, T.K. Bose, Magnetic measurements: A powerful tool in magnetic refrigerator design, Journal of applied physics 77(7) (1995) 3528-3537.

[47] R. Goetzinger, M. Barth, D. Herlach, Growth of lamellar eutectic dendrites in undercooled melts, Journal of applied physics 84(3) (1998) 1643-1649.

[48] J. Li, Y. Zhou, Eutectic growth in bulk undercooled melts, Acta materialia 53(8) (2005) 2351-2359. 
[49] T. Krenke, X. Moya, S. Aksoy, M. Acet, P. Entel, L. Manosa, A. Planes, Y. Elerman, A. Yucel, E.F. Wassermann, Electronic aspects of the martensitic transition in Ni-Mn based Heusler alloys, Journal of Magnetism and Magnetic Materials 310(2, Part 3) (2007) 2788-2789. [50] Y. Sutou, Y. Imano, N. Koeda, T. Omori, R. Kainuma, K. Ishida, K. Oikawa, Magnetic and martensitic transformations of $\operatorname{NiMnX}(\mathrm{X}=\mathrm{In}, \mathrm{Sn}, \mathrm{Sb})$ ferromagnetic shape memory alloys, Applied Physics Letters 85(19) (2004) 4358-4360.

[51] C. Lin, H. Yan, Y. Zhang, C. Esling, X. Zhao, L. Zuo, Crystal structure of modulated martensite and crystallographic correlations between martensite variants of Ni50Mn38Sn12 alloy, Journal of Applied Crystallography 49(4) (2016) 1276-1283.

[52] D.H. Bacon, L. Edwards, J.E. Moffatt, M.E. Fitzpatrick, Synchrotron X-ray diffraction measurements of internal stresses during loading of steel-based metal matrix composites reinforced with TiB2 particles, Acta Materialia 59(9) (2011) 3373-3383.

[53] M.L. Young, J. DeFouw, J.D. Almer, D.C. Dunand, Load partitioning during compressive loading of a Mg/MgB2 composite, Acta Materialia 55(10) (2007) 3467-3478.

[54] P.F. Becher, Microstructural Design of Toughened Ceramics, Journal of the American Ceramic Society 74(2) (1991) 255-269.

[55] R. Becker, W. Döring, Ferromagnetismus, Springer-Verlag2013.

[56] X. Chen, V.B. Naik, R. Mahendiran, R.V. Ramanujan, Optimization of Ni-Co-Mn-Sn Heusler alloy composition for near room temperature magnetic cooling, Journal of Alloys and Compounds 618 (2015) 187-191.

[57] T. Gottschall, K.P. Skokov, R. Burriel, O. Gutfleisch, On the S(T) diagram of magnetocaloric materials with first-order transition: Kinetic and cyclic effects of Heusler alloys, Acta Materialia 107 (2016) 1-8.

[58] K. Gschneidner Jr, V. Pecharsky, A. Pecharsky, C. Zimm, Recent developments in magnetic refrigeration, Materials science forum, Trans Tech Publ, 1999, pp. 69-76.

[59] J. Brock, M. Khan, Large refrigeration capacities near room temperature in Ni2Mn1-xCrxIn, Journal of Magnetism and Magnetic Materials 425 (2017) 1-5.

[60] Y.Q. Ma, C.B. Jiang, G. Feng, H.B. Xu, Thermal stability of the Ni54Mn25Ga21 Heusler alloy with high temperature transformation, Scripta Materialia 48(4) (2003) 365-369.

[61] J. Zhang, S. Hao, D. Jiang, Y. Huan, L. Cui, Y. Liu, Y. Ren, H. Yang, Dual-phase synergy enabled large elastic strains of nanoinclusions in a dislocation slip matrix composite, Nano letters 18(5) (2018) 2976-2983.

[62] Y. Wu, X. Wang, J. Wang, C. Jiang, H. Xu, Magneto-structural transition and magnetocaloric effect of melt spinning Ni50Mn29Ga21-xTbx ( $\mathrm{x}=0-1)$ ribbons, Intermetallics 69 (2016) 118-122.

[63] Y. Aydogdu, A. Turabi, A. Aydogdu, M. Kok, Z. Yakinci, H. Karaca, The effects of boron addition on the magnetic and mechanical properties of NiMnSn shape memory alloys, Journal of Thermal Analysis and Calorimetry 126(2) (2016) 399-406.

[64] Y. Zhang, J. Liu, Q. Zheng, J. Zhang, W. Xia, J. Du, A. Yan, Large magnetic entropy change and enhanced mechanical properties of Ni-Mn-Sn-C alloys, Scripta Materialia 75 (2014) 26-29.

[65] Q. Shen, D. Zhao, W. Sun, Y. Li, J. Liu, The effect of Tb on elastocaloric and mechanical properties of Ni-Mn-In-Tb alloys, Journal of Alloys and Compounds 696 (2017) 538-542. 
[66] C. Tan, K. Zhang, X. Tian, W. Cai, Effect of Gd addition on microstructure, martensitic transformation and mechanical properties of Ni50Mn36Sn14 ferromagnetic shape memory alloy, Journal of Alloys and Compounds 692 (2017) 288-293.

[67] Z. Zhou, L. Yang, R. Li, J. Li, Q. Hu, J. Li, Martensite transformation, mechanical properties and shape memory effects of Ni-Mn-In-Mg shape memory alloys, Progress in Natural Science: Materials International 28(1) (2018) 60-65.

[68] B. Tian, Y. Jiang, F. Chen, Y. Tong, L. Li, Y. Zheng, Effect of Zr addition on the microstructure, phase transformation and mechanical property of Ni50Mn25Ga17Cu8 alloy, Materials Science and Engineering: A 617 (2014) 46-51.

[69] F. Heredia, M. He, G. Lucas, A. Evans, H. Deve, D. Konitzer, The fracture resistance of directionally solidified dual-phase $\mathrm{NiAl}$ reinforced with refractory metals, Acta metallurgica et materialia 41(2) (1993) 505-511.

[70] R. Maaß, S. Van Petegem, D. Ma, J. Zimmermann, D. Grolimund, F. Roters, H. Van Swygenhoven, D. Raabe, Smaller is stronger: The effect of strain hardening, Acta Materialia 57(20) (2009) 5996-6005.

[71] J.R. Greer, J.T.M. De Hosson, Plasticity in small-sized metallic systems: Intrinsic versus extrinsic size effect, Progress in Materials Science 56(6) (2011) 654-724.

[72] J.R. Greer, W.C. Oliver, W.D. Nix, Size dependence of mechanical properties of gold at the micron scale in the absence of strain gradients, Acta Materialia 53(6) (2005) 1821-1830.

[73] A.F. Bower, M. Ortiz, A three-dimensional analysis of crack trapping and bridging by tough particles, Journal of the Mechanics and Physics of Solids 39(6) (1991) 815-858.

[74] H. Gao, J.R. Rice, A first-order perturbation analysis of crack trapping by arrays of obstacles, Journal of applied mechanics 56(4) (1989) 828-836. 